\title{
The gauge structure of exceptional field theories and the tensor hierarchy
}

\author{
G. Aldazabal, ${ }^{a, b}$ M. Graña, ${ }^{c}$ D. Marqués ${ }^{d}$ and J.A. Rosabal ${ }^{a, b}$ \\ ${ }^{a}$ Centro Atómico Bariloche, \\ 8400 S.C. de Bariloche, Argentina \\ ${ }^{b}$ Instituto Balseiro (CNEA-UNC) and CONICET, \\ 8400 S.C. de Bariloche, Argentina \\ ${ }^{c}$ Institut de Physique Théorique, CEA/Saclay, \\ 91191 Gif-sur-Yvette Cedex, France \\ ${ }^{d}$ Instituto de Astronomía y Física del Espacio (CONICET-UBA), \\ C.C. 67 - Suc. 28, 1428 Buenos Aires, Argentina \\ E-mail: aldazaba@cab.cnea.gov.ar, mariana.grana@cea.fr, \\ diegomarques@iafe.uba.ar, rosabalj@ib.cnea.gov.ar
}

ABSTRACT: We address the construction of manifest U-duality invariant generalized diffeomorphisms. The closure of the algebra requires an extension of the tangent space to include a tensor hierarchy indicating the existence of an underlying unifying structure, compatible with $E_{11}$ and Borcherds algebras constructions. We begin with four-dimensional gauged maximal supergravity, and build a generalized Lie derivative that encodes all the gauge transformations of the theory. A generalized frame is introduced, which accommodates for all the degrees of freedom, including the tensor hierarchy. The generalized Lie derivative defines generalized field-dependent fluxes containing all the covariant quantities in the theory, and the closure conditions give rise to their corresponding Bianchi Identities. We then move towards the construction of a full generalized Lie derivative defined on an extended space, analyze the closure conditions, and explore the connection with that of maximal gauged supergravity via a generalized Scherk-Schwarz reduction, and with 11-dimensional supergravity.

KEYwords: Flux compactifications, M-Theory, String Duality

ARXIV EPRINT: 1312.4549 


\section{Contents}

1 Introduction 1

2 Setup and summary of main results 4

2.1 Summary of previous results 4

2.2 Summary of new results 6

2.2.1 Generalized diffeomorphisms in gauged maximal supergravity 6

$\begin{array}{lll}\text { 2.2.2 Generalized diffeomorphisms in extended geometry } & 9\end{array}$

3 Generalized diffeomorphisms in gauged maximal supergravity $\quad 10$

$\begin{array}{lll}3.1 & \text { Generalized Lie derivative and closure } & 11\end{array}$

$\begin{array}{lll}3.2 & \text { The next step in the hierarchy }(\mathbf{9 1 2}) & 12\end{array}$

3.3 The next step in the hierarchy $(\mathbf{1 3 3}+\mathbf{8 6 4 5})$ and so on... 14

$\begin{array}{ll}3.4 & \text { The full generalized Lie derivative } \\ 3.5 & 15\end{array}$

$\begin{array}{ll}3.5 \text { Generalized bein } & 17\end{array}$

$\begin{array}{lll}3.6 & \text { Generalized fluxes } & 18\end{array}$

$\begin{array}{ll}3.7 \text { Generalized Bianchi Identities } & 19\end{array}$

4 Generalized diffeomorphisms in extended geometry $\quad 19$

$\begin{array}{lll}4.1 & \text { The canonical extension and the failure of closure } & 19\end{array}$

$\begin{array}{ll}4.2 \text { Including the tensor hierarchy } & 21\end{array}$

4.2.1 The T-duality case: double field theory 22

$\begin{array}{lll}4.2 .2 & \text { The } E_{7(7)} \text { case } & 24\end{array}$

4.2.3 The $E_{n+1(n+1)}$ case for $n<6 \quad 25$

4.3 The gauge structure of the full generalized Lie derivative 29

5 Contact with 11-dimensional supergravity (and beyond) 30

6 Conclusions $\quad 34$

\section{Introduction}

Incorporating stringy symmetries like T-duality into a field theory, or into a (generalized) geometric description led to Double Field Theory (DFT) [1-6] (see [7-9] for reviews and further references) or Generalized Geometry (GG) [10-12] (see [13] for a review). T-duality invariance is realized in DFT by doubling the coordinates of the internal $n$-dimensional compactification space. Namely, besides the usual coordinates conjugate to compact momentum in string toroidal compactifications, a new set of coordinates conjugate to string windings is included. The so-called section condition (or strong constraint) restricts the fields to depend only on half of the double coordinates. In GG the coordinates themselves 
are not doubled, but one considers generalized vectors living on a generalized tangent space with twice the dimension of the ordinary tangent space. Thus, for $n$ compact dimensions, vector fields in DFT or GG span a vector representation of the full T-duality group $\mathrm{O}(n, n)$. A positive definite metric on this space can be defined in terms of the massless states in the NSNS sector of the superstring. Enlarging the diffeomorphism symmetry to include the gauge transformation of the two-form lead to consider a generalized diffeomorphic transformation, encoded in a generalized Lie derivative [1-6, 14].

More generally, promoting U-duality to a symmetry requires a further extension of the double tangent space into an extended or exceptional generalized space [15, 16], or in the spirit of DFT enlarging the compact space itself into a mega-space (a mega-torus [1719] in the case of toroidal backgrounds) with derivatives spanning a representation of the U-duality group. The U-duality symmetry groups in question are the exceptional groups $E_{n+1(n+1)}$ of toroidal compactifications, where $n$ is the dimension of the compactification space in String Theory ( $n+1$ in M-theory). An internal positive definite metric can be defined and parameterized in terms of the degrees of freedom of Type II or M-theory. In this case the diffeomorphisms and gauge transformations are encoded in an extended generalized Lie derivative [20-22].

Interestingly enough, in DFT or GG it is possible to include not only the symmetries corresponding to the $n$ compact dimensions, but also those of the $d$ space-time dimensions. Namely, the full tangent space is doubled, and full $\mathrm{O}(D, D)(D=n+d)$ covariant generalized diffeomorphisms can be constructed. This proves to be a useful unified description, where afterwards, the $d$-dimensional space-time can be decompactified, amounting in DFT to disregard the dual space-time coordinates, leaving a theory with $\mathrm{GL}(d) \times \mathrm{O}(n, n)$ symmetry. In particular, after a generalized Scherk-Schwarz reduction, such theories were shown to lead to the electric bosonic sector of $d$-dimensional half-maximal gauged supergravities [23, 24].

U-duality $E_{n+1(n+1)}$ invariant constructions at the full $D$-dimensional level are tricky. The simplest setups consider only the internal sector, and therefore correspond to truncations of a full Exceptional Generalized Geometry or Exceptional Field Theory (EFT). Restricted to this sector, constructions of generalized Lie derivatives for dimensions $n \leq 6$ can be found in [20-22], and invariant actions in [25-27]. The $n=7$ case is discussed in $[28,29]$, and requires the introduction of the 11-dimensional dual graviton. For dimensions $n \geq 8$, the groups in question are not even finite. A description of gauged maximal supergravities in $d=2$ with $n=8$ covariance is available in [30]. The situation is far less clear for $n>8$, though there are indications pointing to an $E_{11}$ underlying structure [31-37] or Borcherds algebras-based constructions [38]-[41]. Some of these constructions for $n \leq 6$ were explored in the context of generalized Scherk-Schwarz compactifications in [42-44], related to generalized geometric notions in [20, 21, 44-46], and related to F-theory [47].

A key question is how to couple the internal sector discussed above with the external $d$-dimensional space-time. Previous works in this direction are $[48,49]$ and more recently an $E_{6(6)}$ invariant EFT was presented in [50,51]. In the present article we perform a step forward towards a unified description of gauge transformations in terms of a generalized Lie derivative. 
We begin with the simpler setup of generalized Scherk-Schwarz reductions in $d=4$ and $n=6$ which lead to 4 -dimensional gauged $\mathcal{N}=8$ supergravity [52], in which the gaugings [53] are obtained from twists of an internal 56-dimensional parallelizable megaspace [44]. The methodology can be easily adapted to other groups and dimensions. The extended Lie derivative that encodes all the gauge transformations of the reduced theory is one of the central results of the present article. It is constructed from a careful study of closure of the extended diffeomorphism algebra, and it requires extending the $4+56$ tangent space into a larger $E$-tangent space that accommodates all the $p$-form hierarchy [54]-[58]. We pay special attention to the role played in the closure of the diffeomorphism algebra by the so-called intertwining tensors, built out of the embedding tensor.

A generalized vector on the full $E$-tangent space contains 4 components that generate diffeomorphisms in the "external" space, 56 components that generate diffeomorphisms in the generalized "internal" space (which contain the gauge transformations of the vector fields), and extra components that generate the gauge transformations of the $p$-form fields in maximal gauged supergravity. A field-dependent generalized frame for a generalized $E$-vector can then be parameterized by a 4 -dimensional bein, 56 one-form gauge fields, the scalar coset matrix, and extra space-time $p$-forms that carry internal indices in the modules of the so-called tensor hierarchy. By evaluating the generalized Lie derivative on generalized frames, a set of extended dynamical fluxes can be derived. We show that these fluxes contain all form field strengths of 4-dimensional gauged maximal supergravity, and agree with those found in the breaking of $E_{11}$ into GL(4) $\times E_{7(7)}$ [37]. Moreover, the closure conditions for the generalized fluxes reproduce the Bianchi Identities for the curvatures.

Based on the lessons learned in the compactified case, we then move to the general case with no compactification ansatz assumed. Here, we begin general and do not specify the U-duality group. For any U-duality group $E_{n+1(n+1)}$, the generalized coordinates contain $d$ "external" components $x^{\mu}$, and the $n+1$ "internal" coordinates are embedded in a given representation of $E_{n+1(n+1)}$, to achieve duality covariance. The distinction between internal and external is only formal since no compactification is assumed. At any stage a section condition can be imposed that selects an $n+1$ section of the generalized internal space, allowing to make contact with the $d+n+1=11$-dimensional space-time of 11-dimensional supergravity, or $d+n=10$ of type II theories. We see that the intertwining (embedding) tensors uplift to differential intertwining operators and analyze the role played by them in closure. We comment on the relation between the full generalized Lie derivative, and the connection to the 4-dimensional one upon a generalized Scherk-Schwarz reduction. Intriguingly, we identify seemingly obstructions in the construction of fully covariant generalized diffeomorphisms, and suggest how to circumvent them for the different U-duality groups.

The paper is organized as follows. In section 2 we present the setup and the main results of the paper. In section 3 we show how to include the tensor hierarchy in the generalized Lie derivative for the reduced theory, and analyze the role of the intertwiners and $p$-forms in the closure of the gauge algebra. We also give the explicit form of the generalized frame, parameterized in terms of the degrees of freedom of gauged maximal supergravity, and show that we reproduce the corresponding gauge transformations by acting with the generalized diffeomorphism. This section includes the computation of generalized fluxes 
and Bianchi Identities. In section 4, we give a first step towards the construction of a universal (namely, valid for any exceptional duality group) covariant full generalized Lie derivative in the extended space, and explore the closure of the algebra. Concentrating on the case of $E_{7(7)}$, we make contact in section 5 with 11-dimensional supergravity by breaking $E_{7(7)}$ into SL(8) and then further GL(7), where the latter acts on the ordinary "internal" tangent space. We first show how the fields assemble into $E_{7(7)}$ representations. As we go up in the tensor hierarchy, we need to go further and further beyond supergravity and include more and more non-geometric objects ("U" or "exotic" branes [59]-[61]) to fill up $E_{7(7)}$ representations. We then restrict to conventional 11-dimensional supergravity, construct the generalized frame and recover from its generalized Lie derivative the gauge transformations of 11-dimensional supergravity. We conclude in section 6.

\section{Setup and summary of main results}

In this section we briefly review some of the main results of this paper. First we present some developments that appeared recently in the literature, related to generalized diffeomorphisms in the internal space, which serve for a base to the extensions considered in this article. Then we summarize our main results.

\subsection{Summary of previous results}

Recently, U-duality covariant generalized diffeomorphisms for the internal sector of maximal supergravity were considered in $[20,21]$ and [22]. In order to realize manifest $E_{n+1(n+1)}$ invariance, the internal space can be extended to coincide with the dimension of a given representation of the U-duality group.

Noting the internal derivatives as $\partial_{M}$, the covariant exceptional or extended Dorfman bracket takes the general structure

$$
\left(\mathcal{L}_{\hat{\xi}} \hat{V}\right)^{M}=\hat{\xi}^{P} \partial_{P} \hat{V}^{M}-\hat{V}^{P} \partial_{P} \hat{\xi}^{M}+Y^{M}{ }_{N}{ }^{P}{ }_{Q} \partial_{P} \hat{\xi}^{Q} \hat{V}^{N}
$$

where $\hat{\xi}$ and $\hat{V}$ are vectors in the same representation than the coordinates. We are using the notation that hatted quantities depend on all the internal and external coordinates, although at this stage the external dependence is not important. This generalized Lie derivative is the internal one, and has to be distinguished from the full generalized Lie derivative to be constructed later, which will be hatted. The tensor $Y$ depends on the particular U-duality group invariants, and measures the deviation from standard Riemannian geometry governed by the first two terms above, which correspond to the Lie derivative in the internal sector. We give its components for $E_{n+1(n+1)}$ with $n \leq 5$ in (4.35), and for $E_{7(7)}$ in (2.7).

Closure of the generalized Lie derivative is not automatic, and requires imposing closure constraints. These constraints can be solved by imposing a "section condition" that restricts the theory further $[20,21] .{ }^{1}$ This condition implies that the following two opera-

\footnotetext{
${ }^{1}$ The section condition states that $P^{M N}{ }_{P Q} \partial_{M} \otimes \partial_{N}=0$ (where $P$ is the projector to the second module of the duality group) must annihilate any field or gauge parameter, and also products of them.
} 
tors vanish when acting on any product of fields and/or gauge parameters

$$
\begin{aligned}
& Y^{M}{ }_{P}{ }_{Q}{ }_{Q} \partial_{M} \otimes \partial_{N}=0 \\
& \left(Y^{M}{ }_{Q}{ }_{P} Y^{P}{ }_{R}^{T}{ }_{S}-Y^{M}{ }_{R}{ }^{N} \delta_{Q}^{T}\right) \partial_{(N} \otimes \partial_{T)}=0
\end{aligned}
$$

In the paper we will not assume these constraints, but they can be implemented at any stage.

Closure requires actually more relaxed constraints. In particular, in the context of generalized Scherk-Schwarz configurations [42-44], the section condition was proved to be too strong [62,63], and only a subset of gauged supergravities can be obtained upon dimensional reduction when the framework is restricted by it. In contrast, closure constraints allow for solutions that violate the strong constraint, which permit to make contact with all the admissible deformations of the theory (see for example [42-44, 64] for more details). Twisting the generalized Lie derivative (2.1) with a U-duality valued twist matrix $U_{A}{ }^{M}$ leads to the gaugings $F_{A B}{ }^{C}$

$$
F_{A B}{ }^{C}=2 \Omega_{[A B]}{ }^{C}+Y^{C}{ }_{B}{ }_{E} \Omega_{D A}{ }^{E}, \quad \Omega_{A B}{ }^{C}=U_{A}{ }^{M} \partial_{M} U_{B}{ }^{N}\left(U^{-1}\right)_{N}{ }^{C}
$$

which automatically satisfy the linear constraints of gauged maximal supergravity, projecting out the representations not allowed by supersymmetry. In addition, the closure constraints force the gaugings to satisfy the quadratic constraints of gauged maximal supergravity

$$
F_{A D}^{E} F_{B E}^{F}-F_{B D}^{E} F_{A E}^{F}+F_{A B}^{E} F_{E D}^{F}=0
$$

While the antisymmetric part takes the form of a Jacobi Identity, the symmetric part is not automatically satisfied and depends on the symmetric part of the gaugings $F_{(A B)}{ }^{E}$, called the intertwining tensor, which vanishes under the following contraction

$$
F_{(A B)}{ }^{E} F_{E D}{ }^{F}=0
$$

due to the symmetrization of (2.5).

Let us now specialize to the $E_{7(7)}$ case, which will be the case we explore in section 3. In a previous paper [44] we have addressed the construction of an extended geometry for the internal sector of 4-dimensional maximal gauged supergravity. In order to realize manifest $E_{7(7)}$ invariance, the internal tangent space was taken to be 56-dimensional, in accordance with the dimensionality of the fundamental 56 representation of $E_{7(7)}$. In this case, the group must be augmented with an $\mathbb{R}^{+}$factor, necessary for closure of the algebra, as explained in $[20,21]$. There are two $E_{7(7)}$ invariants, a symplectic metric $\omega_{M N}$ (which raises and lowers indices) and the projector to the adjoint 133 representation $P^{M}{ }_{N}{ }^{P}{ }_{Q}$. In terms of them, the $Y$-tensor takes the form

$$
Y^{M}{ }_{N}{ }_{Q}=-12 P^{M P}{ }_{N Q}+\frac{1}{2} \omega^{M P} \omega_{N Q}
$$

Performing a twist in terms of an internal index-valued frame $\hat{E}_{\bar{A}}{ }^{M}$, we found the expression for the internal generalized fluxes generated by the corresponding mega-twisted-torus

$$
\mathbb{F}_{\bar{A} \bar{B}}^{\bar{C}}=2 \Omega_{[\bar{A} \bar{B}]}^{\bar{C}}+Y^{\bar{C}}{ }_{\bar{B}}^{\bar{D}}{ }_{\bar{E}} \Omega_{\bar{D} \bar{A}}^{\bar{E}}, \quad \Omega_{\bar{A} \bar{B}}{ }^{\bar{C}}=\hat{E}_{\bar{A}}{ }^{M} \partial_{M} \hat{E}_{\bar{B}}{ }^{N}\left(\hat{E}^{-1}\right)_{N}{ }^{\bar{C}}
$$


Although the above analysis was restricted purely to the internal sector, in the particular case of a generalized Scherk-Schwarz compactification the frame decomposes as

$$
\hat{E}_{\bar{A}}^{M}(x, Y)=\Phi_{\bar{A}}^{B}(x) U_{B}^{M}(Y)
$$

with $\Phi_{\bar{A}}{ }^{B}(x)$ containing the scalar fields and $U_{B}{ }^{M}(Y)$ the twist matrix that generates the gaugings in the reduced theory. Since the scalars depend on the 4-dimensional space-time coordinates $x^{\mu}$, with $\mu=1, \ldots, 4$, they were regarded as constants form the internal sector point of view. Then, after a Scherk-Schwarz reduction, the above fluxes can be cast in the form

$$
\mathbb{F}_{\bar{A} \bar{B}}^{\bar{C}}(x)=\Phi_{\bar{A}}^{A} \Phi_{\bar{B}}^{B}\left(\Phi^{-1}\right)_{C}^{\bar{C}} F_{A B}^{C}
$$

where $F_{A B}{ }^{C}$ are taken to be constant and identified with the gaugings of maximal supergravity. It can be checked that by construction they belong to the $\mathbf{5 6}+\mathbf{9 1 2}$ representations, and then automatically satisfy the linear constraints of the theory. Finally we note that we are distinguishing between three types of indices: $M, N, \ldots$ refer to curved internal indices in the extended theory, $A, B, \ldots$ refer to curved internal indices in the reduced theory, and $\bar{A}, \bar{B}, \ldots$ are the flat indices in both.

\subsection{Summary of new results}

In this paper we explore how to extend the above construction by coupling the missing space-time dimensions. We will start with what we will call the "compactified" case, in which we assume a Scherk-Schwarz-type ansatz, with $Y$-independent fluxes of the form (2.10). This case leads to 4-dimensional maximal gauged supergravity. Later, we will extend most of our results to the "decompactified" case, i.e. where we assume that the generalized tangent space splits into 4 and 56 (or actually more, as we will see) directions, but where everything depends in a generic way on external and internal coordinates.

We begin with the compactified case, specializing to the $E_{7(7)}$ U-duality group, and explore the role of the intertwining tensors and the tensor hierarchy in the closure of the algebra. We will extract lessons that will help in building a full generalized Lie derivative in the extended space.

\subsubsection{Generalized diffeomorphisms in gauged maximal supergravity}

Let us begin with the 4-dimensional case of gauged maximal supergravity, with U-duality group $E_{7(7)}$. All the expressions found here coincide with the results obtained in the gauged maximal supergravity formulation of [52], the $E_{11}$ approach in [31-36]-[37] or Borcherds algebras constructions [38]-[41]. The generalized $E$-vector fields (in particular gauge parameters) are of the form

$$
\begin{aligned}
\xi^{\mathbb{A}} & =\left(\xi^{\mu}, \xi^{A}, \xi_{\mu}<A B>, \xi_{\mu \nu}<A B C>, \xi_{\mu \nu \rho}<A B C D>, \ldots\right) \\
& =\left(\xi^{\mu}, \xi^{A}, \xi_{\mu}{ }^{\alpha}, \xi_{\mu \nu}{ }^{\mathcal{A}}, \xi_{\mu \nu \rho}{ }^{\mathbf{A}}, \ldots\right)
\end{aligned}
$$

where $A, B, C$ are indices in the first module of the duality group (for $E_{6(6)}$ and $E_{7(7)}$, this corresponds to the fundamental representation), and $\langle\cdots\rangle$ means a projection from the 
tensor product of various fundamental indices to a particular irreducible representation (or sums of irreducible representations), labeled by $\alpha, \mathcal{A}, \mathbf{A}, \ldots$ on the second line. In the case of $E_{7(7)}, A$ is a fundamental $\mathbf{5 6}$ index, $\alpha$ takes values in the adjoint $\mathbf{1 3 3}$ representation, $\mathcal{A}$ belongs to the $\mathbf{9 1 2}$ representation, $\mathrm{A}$ to the $\mathbf{8 6 4 5}+\mathbf{1 3 3}$ representation, and so on. We will comment on the end of this hierarchy in due time. If we think of these as gauge parameters, they include the usual Riemannian geometry diffeomorphisms generated by four-dimensional vectors $\xi^{\mu}$, (extended generalized) diffeomorphisms of the internal space $\xi^{M}$, plus new extra gauge parameters required for the gauge algebra to close. Note that these gauge parameters do not carry a hat, because they only depend on the external coordinates. The general structure of the generalized Lie derivative is given by

$$
\left(\hat{\mathcal{L}}_{\xi_{1}} \xi_{2}\right)^{\mathbb{A}}=\xi_{1}^{\mathbb{B}} \partial_{\mathbb{B}} \xi_{2}^{\mathbb{A}}-\xi_{2}^{\mathbb{B}} \partial_{\mathbb{B}} \xi_{1}^{\mathbb{A}}+W^{\mathbb{A}} \mathbb{B}_{\mathbb{D}} \partial_{\mathbb{C}} \xi_{1}^{\mathbb{D}} \xi_{2}^{\mathbb{B}}+F_{\mathbb{B C}} \xi_{1}^{\mathbb{A}} \xi_{2}^{\mathbb{C}}
$$

where, since we are considering the compactified case here, we have $\partial_{\mathbb{A}}=\left(\partial_{\mu}, 0, \ldots\right)$. Here, we put a hat on the generalized Lie derivative to emphasize that it corresponds to the (compactified) full Lie derivative. In the core of the paper we will give more explicit expression for all these quantities, here we are simply stating the general form of our results. The $W^{\mathbb{A}} \mathbb{B} \mathbb{C}_{\mathbb{D}}$ tensor is formed by GL(4) and $E_{7(7)}$ invariants, and its purely external components vanish in accordance with Riemannian geometry. While the first three terms are un-gauged, the tensor $F_{\mathbb{B} C^{\mathbb{A}}}$ depends linearly on the gaugings, and then carries the information on the internal $Y$-tensor introduced in (2.1) through (2.4).

Out of the gaugings and generators of the group, one builds a hierarchy of intertwining tensors ${ }^{2}$

$$
F_{\alpha}{ }^{A}, \quad F_{\mathcal{A}}{ }^{\alpha}, \quad F_{\mathbf{A}}^{\mathcal{A}}, \quad \ldots
$$

which are such that when a given component of the generalized Lie derivative is projected by its corresponding intertwining tensor, the sub-algebra formed by it, together with the previous components, closes. The reason for this is that the contraction between successive intertwining tensors vanishes

$$
F_{\mathcal{A}}{ }^{\alpha} F_{\alpha}{ }^{A}=F_{\mathbf{A}}{ }^{\mathcal{A}} F_{\mathcal{A}}{ }^{\alpha}=\cdots=0
$$

and the failure of closure when the hierarchy is truncated to a given level, is proportional to the intertwiner at that level.

A field-dependent generalized frame in the $E$-tangent space can be introduced

$$
\mathbb{E}_{\overline{\mathbb{A}}}{ }^{\mathbb{A}}=\left(\begin{array}{ccccc}
e_{\bar{a}}{ }^{\mu}-e_{\bar{a}}{ }^{\rho} A_{\rho}{ }^{A} & -e_{\bar{a}}{ }^{\rho}\left(B_{\rho \mu}{ }^{\alpha}-A_{\rho}{ }^{B} A_{\mu}{ }^{C}\left(t^{\alpha}\right)_{B C}\right) & \mathbb{E}_{\bar{a} \nu \rho}{ }^{\mathcal{A}} \\
0 & \Phi_{\bar{A}}{ }^{M} & -2 A_{\mu}{ }^{B} \Phi_{\bar{A}}{ }^{C}\left(t^{\alpha}\right)_{B C} & \cdots & \\
0 & 0 & -\left(e^{-1}\right)_{\mu}{ }^{\bar{a}}\left(t_{\bar{\alpha}}\right)^{\bar{A} \bar{B}} \Phi_{\bar{A}}{ }^{A} \Phi_{\bar{B}}{ }^{B}\left(t^{\alpha}\right)_{A B} & \cdots & \\
& & & & \ddots
\end{array}\right)
$$

where, in particular

$$
\begin{aligned}
\mathbb{E}_{\bar{a} \nu \rho}^{\mathcal{A}}=-e_{\bar{a}}^{\mu}\left[C_{\mu \nu \rho}{ }^{\mathcal{A}}+\right. & S^{\mathcal{A}}{ }_{A \alpha} A_{\mu}{ }^{A} B_{\nu \rho}{ }^{\alpha} \\
& \left.-\frac{1}{3} S^{\mathcal{A}}{ }_{A \alpha}\left(t^{\alpha}\right)_{B C}\left(A_{\mu}{ }^{A} A_{\nu}{ }^{B} A_{\rho}{ }^{C}+2 A_{\mu}{ }^{B} A_{\nu}{ }^{C} A_{\rho}{ }^{A}\right)\right]
\end{aligned}
$$

\footnotetext{
${ }^{2}$ For example, $F_{\alpha}{ }^{A}=\left(t_{\alpha}\right)^{B C} F_{(B C)}{ }^{A}$.
} 
contains the 3 -form fields (and $S^{\mathcal{A}} A \alpha$ is the projector from the $\mathbf{5 6} \times \mathbf{1 3 3}$ to the $\mathbf{9 1 2}$ representation, given for example in [37]), and the other components represented by the dots contain the remaining $p$-forms. This allows to make contact with the fields in gauged maximal supergravity, namely a 4-dimensional bein $e_{\bar{a}}{ }^{\mu}$, scalars $\Phi_{\bar{A}}{ }^{A}$, gauge vector fields $A_{\mu}{ }^{A}$, and the (in)famous $p$-form fields that build the tensor hierarchy $B_{\mu \nu}{ }^{\alpha}, C_{\mu \nu \rho} \mathcal{A}, \ldots$ Inserting this in the generalized Lie derivative, we obtain the gauge transformations of each field

$$
\begin{aligned}
\hat{\delta}_{\xi} e_{\bar{a}}{ }^{\mu}= & L_{\xi} e_{\bar{a}}^{\mu} \\
\hat{\delta}_{\xi} A_{\mu}{ }^{A}= & L_{\xi} A_{\mu}{ }^{A}+\partial_{\mu} \xi^{A}+F_{B C}{ }^{A} \xi^{B} A_{\mu}{ }^{C}-\xi_{\mu}{ }^{A} \\
\hat{\delta}_{\xi} \Phi_{\bar{A}}{ }^{A}= & L_{\xi} \Phi_{\bar{A}}{ }^{A}+F_{B C}{ }^{A} \xi^{B} \Phi_{\bar{A}}{ }^{C} \\
\hat{\delta}_{\xi} B_{\mu \nu}{ }^{\alpha}= & L_{\xi} B_{\mu \nu}{ }^{\alpha}+2 \partial_{[\mu} \xi_{\nu]}^{\alpha}-\xi_{\mu \nu}{ }^{\alpha}+2\left(t^{\alpha}\right)_{B C}\left(A_{[\mu}{ }^{B} \xi_{\nu]}{ }^{C}-A_{[\mu}{ }^{B} \partial_{\nu]} \xi^{C}\right) \\
& -F_{A \beta}{ }^{\alpha} \xi^{A} B_{\mu \nu}{ }^{\beta}-2\left(t^{\alpha}\right)_{B C} \xi^{B} F_{\beta}{ }^{C} B_{\mu \nu}{ }^{\beta}
\end{aligned}
$$

(where $L_{\xi}$ is the ordinary 4-dimensional Lie derivative along $\xi^{\mu}$ ) which faithfully reproduce those of gauged maximal supergravity.

When the generalized Lie derivative is evaluated on frames, it defines the generalized fluxes

$$
\mathbb{F}_{\overline{\mathbb{A}} \overline{\mathbb{B}}} \overline{\overline{\mathbb{C}}}=\left(\hat{\mathcal{L}}_{\mathbb{E}_{\overline{\mathbb{A}}}} \mathbb{E}_{\overline{\mathbb{B}}}\right)^{\mathbb{C}}\left(\mathbb{E}^{-1}\right)_{\mathbb{C}}^{\overline{\mathbb{C}}}
$$

whose components determine the covariant quantities of gauged maximal supergravity. We can list some of them

$$
\begin{aligned}
\mathbb{F}_{\bar{a} \bar{b}}^{\bar{c}} & =2 e_{[\bar{a}}^{\rho} \partial_{\rho} e_{\bar{b}]}^{\sigma} e_{\sigma}^{\bar{c}}=\omega_{[\bar{a} \bar{b}]}^{\bar{c}} \\
\mathbb{F}_{\bar{a} \bar{b}} \bar{C} & =-e_{\bar{a}}^{\mu} e_{\bar{b}}^{\nu}\left(\Phi^{-1}\right)_{C} \bar{C} \mathcal{F}_{\mu \nu}{ }^{C} \\
\mathbb{F}_{\bar{a} \bar{b} \bar{c} \bar{c}} & =e_{\bar{a}}^{\mu} e_{\bar{b}}^{\nu} e_{\bar{c}}{ }^{\rho}\left(t_{\alpha}\right)^{A B}\left(\Phi^{-1}\right)_{A}{ }^{\bar{A}}\left(\Phi^{-1}\right)_{B} \bar{B}\left(t^{\bar{\gamma}}\right)_{\bar{A} \bar{B}} \mathcal{H}_{\mu \nu \rho}{ }^{\alpha} \\
\mathbb{F}_{\bar{A} \bar{B}} \bar{C} & =\Phi_{\bar{A}}{ }^{A} \Phi_{\bar{B}}{ }^{B}\left(\Phi^{-1}\right)_{C} \bar{C} F_{A B}{ }^{C} \\
\mathbb{F}_{\bar{a} \bar{B} \bar{C}} \bar{C} & =-\mathbb{F}_{\bar{B} \bar{a}} \bar{C}^{\bar{C}}=e_{\bar{a}}^{\mu}\left(\Phi^{-1}\right)_{C}{ }^{\bar{C}} D_{\mu} \Phi_{\bar{B}}^{C}
\end{aligned}
$$

where

$$
\begin{aligned}
D_{\mu} \Phi_{\bar{B}}{ }^{C} & =\partial_{\mu} \Phi_{\bar{B}}{ }^{C}-F_{A B}{ }^{C} A_{\mu}{ }^{A} \Phi_{\bar{B}}{ }^{B} \\
\mathcal{F}_{\mu \nu}{ }^{C} & =2 \partial_{[\mu} A_{\nu]}{ }^{C}-F_{[A B]}{ }^{C} A_{\mu}{ }^{A} A_{\nu}{ }^{B}+B_{\mu \nu}{ }^{\alpha} F_{\alpha}{ }^{C} \\
\mathcal{H}_{\mu \nu \rho}{ }^{\alpha}=3\left[\partial_{[\mu} B_{\nu \rho]}{ }^{\alpha}-C_{\mu \nu \rho}{ }^{\mathcal{A}} F_{\mathcal{A}}{ }^{\alpha}+2\left(t^{\alpha}\right)_{B C}\left(A_{[\mu}{ }^{B} \partial_{\nu} A_{\rho]}{ }^{C}+A_{[\mu}{ }^{B} B_{\nu \rho]}{ }^{\beta} F_{\beta}{ }^{C}\right.\right. & \\
& \left.\left.+\frac{1}{3} F_{D E}^{B} A_{[\mu}^{D} A_{\nu}{ }^{E} A_{\rho]}{ }^{C}\right)\right]
\end{aligned}
$$

Notice that the internal generalized fluxes that encode the gaugings (2.10) arise here as particular components. Other components are the antisymmetric part of the 4-dimensional spin connection, the curvatures of the 1 and 2 -forms, the covariant derivatives of the scalars, etc. 
Since the generalized Lie derivative forms a closed algebra, and the generalized fluxes are defined in terms of it, the closure conditions correspond to Bianchi Identities (BI)

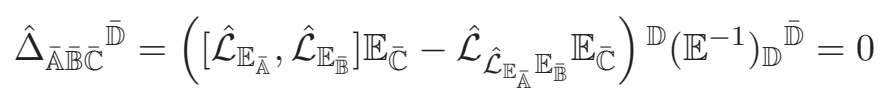

These generalized BI include as particular components those of the four dimensional Riemann tensor and that of the curvature for the two form (for simplicity we compute only the projection of the latter via the corresponding intertwiner)

$$
\begin{aligned}
\hat{\Delta}_{\bar{d} \bar{a} \bar{b}}{ }^{\bar{c}} & =3 e_{\bar{a}}^{\mu} e_{\bar{d}}^{\nu} e_{\bar{b}}^{\rho}\left(e^{-1}\right)_{\sigma}{ }^{\bar{c}} R_{[\mu \nu \rho]}^{\sigma}=3\left(\partial_{[\bar{a}} \omega_{\overline{d b}]}^{\bar{c}}+\omega_{[\bar{a} \bar{d}}{ }^{\bar{e}} \omega_{\bar{b}] \bar{e}}{ }^{\bar{c}}\right) \\
\hat{\Delta}_{\bar{d} \bar{a} \bar{b}} \bar{c} & =e_{\bar{d}}^{\mu} e_{\bar{a}}^{\nu} e_{\bar{b}}^{\rho}\left(\Phi^{-1}\right)_{M} \bar{C}\left(3 D_{[\mu} \mathcal{F}_{\nu \rho]}{ }^{M}-\mathcal{H}_{\mu \nu \rho}{ }^{M}\right)
\end{aligned}
$$

We note that they contain the BIs of General Relativity and those of the gauge sector of maximal supergravity in a unified way.

\subsubsection{Generalized diffeomorphisms in extended geometry}

Next we explore the decompactified case, towards the construction of a full generalized Lie derivative containing derivatives with respect to the external and internal components. The generalized Lie derivative in the four-dimensional case (2.12) takes the form of a "gauged" generalized Lie derivative. Its structure coincides with that of Gauged DFT [65], which can be obtained from a higher-dimensional generalized Lie derivative through a generalized Scherk-Schwarz reduction [62,63]. Here we explore to what extent the gauged generalized Lie derivative (2.12) admits an uplift to an extended space. However, we will be general and not specify a particular U-duality group. Now, the generalized vector fields also span an extended tangent space

$$
\hat{\xi}^{\mathbb{M}}=\left(\hat{\xi}^{\mu}, \hat{\xi}^{M}, \hat{\xi}_{\mu}^{<M N>}, \hat{\xi}_{\mu \nu}<M N P>, \ldots\right)
$$

and moreover, we have put a hat on them to signal that they depend on both external and internal coordinates, and recall the notation $\langle\cdots\rangle$ means a projection to the relevant representations in the tensor hierarchy for the different levels, where we name the level $p \geq 1$ as that of the $p-1$-form gauge parameter.

Schematically, the generalized Lie derivative adopts the following form

$$
\left(\hat{\mathcal{L}}_{\hat{\xi}_{1}} \hat{\xi}_{2}\right) \stackrel{\mathbb{M}}{=} \hat{\xi}_{1}^{\mathbb{P}} \partial_{\mathbb{P}} \hat{\xi}_{2}^{\mathbb{M}}-\hat{\xi}_{2}^{\mathbb{P}} \partial_{\mathbb{P}} \hat{\xi}_{1}^{\mathbb{M}}+Y^{\mathbb{M}} \mathbb{P}_{\mathbb{Q}}^{\mathbb{N}} \partial_{\mathbb{N}} \hat{\xi}_{1}^{\hat{Q}} \hat{\xi}_{2}^{\mathbb{P}}
$$

where derivatives now act with respect to internal directions also $\partial_{\mathbb{M}}=\left(\partial_{\mu}, \partial_{M}, 0, \ldots\right)$. One could also consider derivatives with respect to the extended tangent directions associated to the $p$-forms, and constrain them through a generalized section condition, but this is not the approach we adopt here. The generalized $Y$-tensor contains $\operatorname{GL}(d)$ and $E_{n+1(n+1)}$ invariants, and when it is restricted to the internal sector it coincides with the $Y$-tensors of the different U-duality groups, in particular with (2.7) for $E_{7(7)}$. The purely external components of it vanish, and then one recovers the external diffeomorphisms of Riemannian geometry. The details will be presented in section 4 . The generalized $Y$-tensor is the one 
that projects the components of the generalized vectors to the relevant representation according to the U-duality group and the level of the hierarchy.

Performing a generalized Scherk-Schwarz reduction $\hat{\xi}^{\mathbb{M}}=U_{\mathbb{A}} \mathbb{M}^{\mathbb{M}}(Y) \xi^{\mathbb{A}}(x)$, and plugging it above, one can make contact with the gauged generalized Lie derivative (2.12). When the internal derivatives $\partial_{M}$ hit the twist matrix $\mathrm{U}(Y)$, it forms gaugings, that are contained in the last term in (2.12). On the other hand, when the external derivatives $\partial_{\mu}$ hit the $x$-dependent part, this reproduces the first three terms in (2.12). Then, the $W$-tensor there is the generalized $Y$-tensor here.

We have worked the hierarchy up to the 2-form level, and found a couple of intriguing facts. To begin with, the first level component $\left(\hat{\mathcal{L}}_{\hat{\xi}_{1}} \hat{\xi}_{2}\right)^{M}$ contains terms that are projected by the first "intertwining" operator ${ }^{3}$

$$
\frac{1}{2} Y^{M}{ }_{P}^{N}{ }_{Q} \partial_{N}(\ldots)^{P Q}
$$

and closes up to section condition-like terms (that compactify to quadratic constraints) and terms proportional to $Y^{M}{ }_{\left[P^{N}\right.}{ }_{Q]}$. While this vanishes when the U-duality group is $E_{n+1(n+1)}$ with $n<6$, we find a closure obstruction for $E_{7(7)}$ already at the first level of the hierarchy. ${ }^{4}$ Regarding the second level component $\left(\hat{\mathcal{L}}_{\hat{\xi}} \hat{V}\right)_{\mu}<M N>$, it includes terms that are projected by the second "intertwining" operator

$$
\tilde{Y}^{M N T}{ }_{Q R S} \partial_{T}, \quad \text { where } \tilde{Y}^{M N T}{ }_{Q R S}=Y^{M}{ }_{P}{ }_{Q}{ }_{Q} Y^{P}{ }_{R}^{T}{ }_{S}-Y^{M}{ }_{R}{ }_{S} \delta_{Q}^{T}
$$

The closure of this component is proportional to terms that depend on $\tilde{Y}^{M N T}{ }_{(Q R S)}$. While this vanishes for $n<5$, we now find an obstruction for $E_{6(6)}$. It then appears to be a pattern affecting the $E_{n+1(n+1)}$ duality groups at the level $7-n$ of the tensor hierarchy. A discussion on this point can be found in section 4 .

Finally, we have introduced a field-dependent generalized frame, and from it defined the fluxes and extracted the gauge transformations of its components. And we have also connected the tensor hierarchy with M-theory brane charges, and shown how our results reproduce the gauge transformations of the fields in 11-dimensional supergravity.

\section{Generalized diffeomorphisms in gauged maximal supergravity}

Our construction will begin with the canonical generalized Lie derivative in a Yang-Mills theory coupled to gravity after a Kaluza-Klein decomposition. Since in our case of interest the gaugings are not strictly speaking structure constants (in this section we focus on the 4-dimensional $E_{7(7)}$ case), i.e. are not antisymmetric, the original proposal will fail to close and require an extension. We will go through this extension in a systematic way, ending with a closed form of a generalized Lie derivative for gauged maximal supergravity. This analysis will serve as a base for the next extension, pointing towards a full generalized Lie derivative in the mega-space-time with manifest U-duality covariance.

\footnotetext{
${ }^{3}$ Notice that when $\frac{1}{2} Y^{M}{ }_{P}{ }^{N}{ }_{Q} \partial_{N}(\ldots)^{P} Q$ acts on twist matrices it is proportional to the intertwining tensor, i.e. $\frac{1}{2} Y^{M}{ }_{P}{ }^{N}{ }_{Q} \partial_{N}\left(U_{A}{ }^{P} U_{B}{ }^{Q}\right)=F_{(A B)}{ }^{C} U_{C}{ }^{M}$. This happens only when the $Y^{M}{ }_{P}{ }^{N}{ }_{Q}$ is symmetric in the $P Q$ indices.

${ }^{4}$ In $E_{8(8)}$ the failure of closure appears already in the purely internal sector, even before coupling it to space-time [22].
} 


\subsection{Generalized Lie derivative and closure}

We begin with a generalized Lie derivative, with the following components

$$
\begin{aligned}
\left(\hat{\mathcal{L}}_{\xi_{1}} \xi_{2}\right)^{\mu} & =\left(L_{\xi_{1}} \xi_{2}\right)^{\mu} \\
\left(\hat{\mathcal{L}}_{\xi_{1}} \xi_{2}\right)^{A} & =L_{\xi_{1}} \xi_{2}^{A}-\xi_{2}^{\rho} \partial_{\rho} \xi_{1}^{A}+F_{B C}{ }^{A} \xi_{1}^{B} \xi_{2}^{C}
\end{aligned}
$$

where $L_{\xi_{1}}$ generate 4 -dimensional diffeomorphisms $\mu=1, \ldots 4$, and the extra components generate gauge transformations (with gauge parameters $\xi^{A}$ ) $A=1, \ldots, 56$. The constant gaugings $F_{A B}{ }^{C}$ belong to the $\mathbf{5 6}+\mathbf{9 1 2}$ representations allowed by the linear supersymmetric constraint in gauged maximal supergravity (see section 2).

Let us briefly state what the closure condition is. We are using the convention that the generalized Lie derivative $\hat{\mathcal{L}}$ acts on objects assuming that they are generalized tensors. We can also define a generalized gauge transformation $\hat{\delta}$ that transforms objects without assuming any covariancy properties. Let us consider an example to understand the difference. While the generalized Lie derivative $\hat{\mathcal{L}}$ treats $\partial_{\mathbb{A}} V^{\mathbb{B}}$ as if it were a tensor (we emphasize that this is mere notation, since the generalized Lie derivative is only defined to act on tensors), the gauge transformation $\hat{\delta}$ commutes with the derivative, transforming this as $\hat{\delta}_{\xi}\left(\partial_{\mathbb{A}} V^{\mathbb{B}}\right)=\partial_{\mathbb{A}}\left(\hat{\delta}_{\xi} V\right)^{\mathbb{B}}=\partial_{\mathbb{A}}\left(\hat{\mathcal{L}}_{\xi} V\right)^{\mathbb{B}}$. Then, we can define the operator

$$
\hat{\Delta}_{\xi}=\hat{\delta}_{\xi}-\hat{\mathcal{L}}_{\xi}
$$

which measures the failure of the covariance of the object on which it acts. So, for example, we have that on vectors $\hat{\Delta}_{\xi} V=0$. In particular, we would want the generalized Lie derivative to transform vectors into vectors, this is the requirement known as closure constraint

$$
\left(\hat{\Delta}_{\xi_{1}} \hat{\mathcal{L}}_{\xi_{2}} V\right)^{\mathbb{A}}=\left[\left(\left[\hat{\mathcal{L}}_{\xi_{1}}, \hat{\mathcal{L}}_{\xi_{2}}\right]-\hat{\mathcal{L}}_{\hat{\mathcal{L}}_{\xi_{1}} \xi_{2}}\right) V\right]^{\mathbb{A}}=0
$$

Then, for the generalized Lie derivative (3.1), the closure conditions become

$$
\begin{aligned}
& \left(\hat{\Delta}_{\xi_{1}} \hat{\mathcal{L}}_{\xi_{2}} V\right)^{\mu}=0 \\
& \left(\hat{\Delta}_{\xi_{1}} \hat{\mathcal{L}}_{\xi_{2}} V\right)^{A}=-2 V^{\rho} F_{(B C)}{ }^{A} \partial_{\rho} \xi_{1}^{B} \xi_{2}^{C}+\left(\left[F_{B}, F_{C}\right]+F_{B C}{ }^{E} F_{E}\right){ }_{D}^{A} \xi_{1}^{B} \xi_{2}^{C} V^{D}
\end{aligned}
$$

Since we are assuming that the gaugings satisfy the quadratic constraints, the last term vanishes, and the failure of the closure is proportional to the symmetric components of the gaugings $F_{(B C)}{ }^{A}$. Symmetrized in this way, the indices $(B C)$ belong to the adjoint 133 representation of $E_{7(7)}$, and $F_{(B C)}{ }^{A}$ is called the intertwining tensor.

We then see that in order to achieve closure, the original generalized Lie derivative has to be extended. Since the failure of closure is proportional to $V^{\mu}$, we can add an additional term

$$
\begin{aligned}
\left(\hat{\mathcal{L}}_{\xi_{1}} \xi_{2}\right)^{\mu} & =\left(L_{\xi_{1}} \xi_{2}\right)^{\mu} \\
\left(\hat{\mathcal{L}}_{\xi_{1}} \xi_{2}\right)^{A} & =L_{\xi_{1}} \xi_{2}^{A}-\xi_{2}^{\rho} \partial_{\rho} \xi_{1}^{A}+F_{B C}{ }^{A} \xi_{1}^{B} \xi_{2}^{C}+\underline{\xi_{2}^{\rho} \xi_{1 \rho}{ }^{A}}
\end{aligned}
$$

containing a new gauge parameter $\xi_{\rho}{ }^{A}$. Its transformation should be such that it cancels the failure of the closure. A quick computation shows that now (we impose the quadratic 
constraints on the gaugings)

$$
\begin{aligned}
\left(\hat{\Delta}_{\xi_{1}} \hat{\mathcal{L}}_{\xi_{2}} V\right)^{\mu} & =0 \\
\left(\hat{\Delta}_{\xi_{1}} \hat{\mathcal{L}}_{\xi_{2}} V\right)^{A} & =V^{\rho}\left[\left(\delta_{\xi_{1}} \xi_{2}\right)_{\rho}{ }^{A}-\left(L_{\xi_{1}} \xi_{2}\right)_{\rho}{ }^{A}-2 \xi_{2}{ }^{\sigma} \partial_{[\sigma} \xi_{1 \rho]}{ }^{A}-2 F_{(B C)}{ }^{A} \partial_{\rho} \xi_{1}^{B} \xi_{2}^{C}\right. \\
& \left.-2 F_{B C}{ }^{A} \xi_{[1}^{B} \xi_{2] \rho}{ }^{C}\right]+F_{B C}{ }^{A} \xi_{2}^{\rho} \xi_{1 \rho}{ }^{B} V^{C}
\end{aligned}
$$

While the first block between brackets dictates how the new gauge parameters must transform, notice that the last term cannot be absorbed in the brackets, and its vanishing must be imposed as a constraint

$$
\xi_{2}^{\rho} \xi_{1 \rho}{ }^{B} F_{B C}{ }^{A} V^{C}=0
$$

Recalling the quadratic constraints we can rapidly find a solution to this equation

$$
\xi_{\mu}{ }^{A}=F_{(B C)}{ }^{A} \xi_{\mu}{ }^{B C}=F_{(B C)}{ }^{A}\left(t_{\alpha}\right)^{B C} \xi_{\mu}{ }^{\alpha}=F_{\alpha}{ }^{A} \xi_{\mu}{ }^{\alpha}
$$

The last step is possible because the intertwining tensor $F_{\alpha}{ }^{A}$ takes values in the adjoint 133 representation of $E_{7(7)}$. Of course, we could have guessed from the beginning that the completion of the original generalized Lie derivative would include components of this form, because the failure for its closure is proportional to the intertwining tensor.

Now, if we generalize the notion of a vector, extending it to include $\xi_{\mu}{ }^{\alpha}$ as new components in an extended tangent space, we now find a closed algebra of the form

$$
\begin{aligned}
\left(\hat{\mathcal{L}}_{\xi_{1}} \xi_{2}\right)^{\mu} & =\left(L_{\xi_{1}} \xi_{2}\right)^{\mu} \\
\left(\hat{\mathcal{L}}_{\xi_{1}} \xi_{2}\right)^{A} & =L_{\xi_{1}} \xi_{2}^{A}-\xi_{2}^{\rho} \partial_{\rho} \xi_{1}^{A}+F_{B C}{ }^{A} \xi_{1}^{B} \xi_{2}^{C}+\xi_{2}^{\rho} \xi_{1 \rho}{ }^{A} \\
\left(\hat{\mathcal{L}}_{\xi_{1}} \xi_{2}\right)_{\mu}{ }^{A} & =\left(L_{\xi_{1}} \xi_{2}\right)_{\mu}{ }^{A}+2 \xi_{2}^{\sigma} \partial_{[\sigma} \xi_{1 \rho]}{ }^{A}+2 F_{(B C)}{ }^{A}\left(2 \xi_{[1}^{B} \xi_{2] \mu}{ }^{C}+\xi_{2}^{B} \partial_{\mu} \xi_{1}^{C}\right)
\end{aligned}
$$

Here the last component is projected by the intertwining tensor from the $\mathbf{1 3 3}$ representation to the $\mathbf{5 6}$ representation $\xi_{\mu}{ }^{A}=F_{\alpha}{ }^{A} \xi_{\mu}{ }^{\alpha}$ as in (3.8). Removing this projection is the topic of the next subsection. By now we have found a closed (projected) generalized Lie derivative, that is enough to reproduce the gauge transformation of the bosonic sector of maximal gauged supergravity in the formulation of [52]. In fact, after some algebra one finds

$$
\left(\hat{\Delta}_{\xi_{1}} \hat{\mathcal{L}}_{\xi_{2}} V\right)_{\mu}{ }^{A}=0
$$

and the full closure is guaranteed.

\subsection{The next step in the hierarchy (912)}

It follows from (3.8) that the last component of the generalized vectors are projected, and thus so is the last component of the generalized Lie derivative. The projection is due to the intertwining tensor

$$
F_{\alpha}{ }^{A}=\left(t_{\alpha}\right)^{B C} F_{(B C)}{ }^{A}
$$

We can then factorize it, and determine the un-projected components up to terms that vanish due to the projection

$$
\begin{aligned}
F_{\alpha}{ }^{A}\left[\left(\hat{\mathcal{L}}_{\xi_{1}} \xi_{2}\right)_{\mu}{ }^{\alpha}=\right. & \left(L_{\xi_{1}} \xi_{2}\right)_{\mu}{ }^{\alpha}+2 \xi_{2}^{\sigma} \partial_{[\sigma} \xi_{1 \mu]}{ }^{\alpha} \\
& \left.-2\left(t^{\alpha}\right)_{B C}\left(2 \xi_{[1}^{B} \xi_{2] \mu}{ }^{\beta} F_{\beta}{ }^{C}+\xi_{2}^{B} \partial_{\mu} \xi_{1}^{C}\right)+\Gamma_{\mu}{ }^{\alpha}\right]
\end{aligned}
$$


Here $\Gamma_{\mu}^{\alpha}$ is the collection of terms that vanish due to the projection, i.e. it satisfies $\Gamma_{\mu}{ }^{\alpha} F_{\alpha}{ }^{A}=0$. Setting for the moment $\Gamma_{\mu}{ }^{\alpha}=0$, we can compute closure of this last un-projected component, finding

$$
\begin{aligned}
\left(\hat{\Delta}_{\xi_{1}} \hat{\mathcal{L}}_{\xi_{2}} \xi_{3}\right)_{\mu}{ }^{\alpha}=-F_{A B C}{ }^{\alpha}[ & \left(2 \xi_{3}^{\rho} \xi_{[1 \rho}{ }^{\gamma} \xi_{2] \mu}{ }^{\beta}-\xi_{3 \mu}{ }^{\gamma} \xi_{2}^{\rho} \xi_{1 \rho}{ }^{\beta}\right) F_{\gamma}{ }^{A}\left(t_{\beta}\right)^{B C} \\
& \left.+2 \xi_{3 \mu}{ }^{\gamma} F_{\gamma}{ }^{C} \xi_{1}^{A} \xi_{2}^{B}+4 \xi_{3}^{B}\left(\xi_{[2}^{A} \xi_{1] \mu}{ }^{\gamma} F_{\gamma}{ }^{C}-\xi_{[2}^{A} \partial_{\mu} \xi_{1]}^{C}\right)\right]
\end{aligned}
$$

Here we have used the quadratic constraints and defined

$$
F_{A B C}^{\alpha}=2\left(F_{A(B}^{D}\left(t^{\alpha}\right)_{C) D}-F_{(B C)}^{D}\left(t^{\alpha}\right)_{D A}\right)
$$

It is easy to see that this tensor satisfies the following properties

$$
P_{B}^{A}{ }^{C}{ }_{D} F_{E C}{ }^{D \alpha}=F_{E B}{ }^{A \alpha}, \quad F_{(A B C)}^{\alpha}=0, \quad F_{A B}^{B \alpha}=F_{B A}^{B \alpha}=0
$$

and then its indices $A(B C)$ belong to the 912 representation of $E_{7(7)}$ [52].

Clearly, since the projected components enjoy a closed algebra, the failure of the unprojected components must cancel through a projection with the intertwining tensor. A short computation shows that

$$
F_{A B C}{ }^{\alpha} F_{\alpha}^{D}=0
$$

due to the quadratic constraints. This makes clear that $\Gamma_{\mu}{ }^{\alpha}$ should be proportional to this tensor. Also, it must be selected so as to cancel the un-projected contributions (3.13). After some algebra we find that the correction to the un-projected generalized Lie derivative is given by

$$
\Gamma_{\mu}^{\alpha}=\xi_{2}^{\rho} \xi_{1 \rho \mu}{ }^{\alpha}-F_{A \beta}{ }^{\alpha} \xi_{2 \mu}{ }^{\beta} \xi_{1}^{A}
$$

Here, we have denoted the indices in the $\mathbf{9 1 2}$ as $A \beta$, and introduced 133 new gauge twoform gauge parameters $\xi_{\rho \mu}{ }^{\alpha}$. However, these are now projected by the new intertwining tensor $F_{A \beta}{ }^{\alpha}$, which projects the $\mathbf{9 1 2}$ into the 133, so this component of the generalized Lie derivative only knows about the projection of the new gauge parameters

$$
\xi_{\mu \nu}{ }^{\alpha}=F_{A \beta}{ }^{\alpha} \xi_{\mu \nu}{ }^{A \beta}
$$

This is analog to the previous intertwining $F_{\alpha}{ }^{A}$, which projects the $\mathbf{1 3 3}$ into the $\mathbf{5 6}$. Introducing (3.17) in (3.12), we find that closure is achieved provided the gauge transformation of the (projection of the) new gauge parameters is given by

$$
\begin{aligned}
& \left(\hat{\mathcal{L}}_{\xi_{1}} \xi_{2}\right)_{\mu \nu}^{\alpha}=\left(L_{\xi_{1}} \xi_{2}\right)_{\mu \nu}^{\alpha}-3 \xi_{2}^{\rho} \partial_{[\rho} \xi_{1 \mu \nu]}^{\alpha}
\end{aligned}
$$

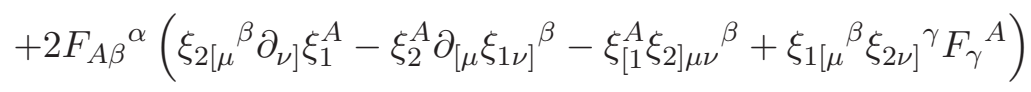


Then, the following algebra closes up to the quadratic constraints

$$
\begin{aligned}
\left(\hat{\mathcal{L}}_{\xi_{1}} \xi_{2}\right)^{\mu}= & \left(L_{\xi_{1}} \xi_{2}\right)^{\mu} \\
\left(\hat{\mathcal{L}}_{\xi_{1}} \xi_{2}\right)^{A}= & L_{\xi_{1}} \xi_{2}^{A}-\xi_{2}^{\rho} \partial_{\rho} \xi_{1}^{A}+F_{B C}{ }^{A} \xi_{1}^{B} \xi_{2}^{C}+\xi_{2}^{\rho} \xi_{1 \rho}{ }^{\gamma} F_{\gamma}{ }^{A} \\
\left(\hat{\mathcal{L}}_{\xi_{1}} \xi_{2}\right)_{\mu}{ }^{\alpha}= & \left(L_{\xi_{1}} \xi_{2}\right)_{\mu}^{\alpha}-2 \xi_{2}^{\rho} \partial_{[\rho} \xi_{1 \mu]}^{\alpha}-2\left(t^{\alpha}\right)_{B C}\left(2 \xi_{[1}^{B} \xi_{2] \mu}{ }^{\gamma} F_{\gamma}{ }^{C}+\xi_{2}^{B} \partial_{\mu} \xi_{1}^{C}\right) \\
& +\xi_{2}^{\rho} \xi_{1 \rho \mu}{ }^{\alpha}-F_{A \beta}{ }^{\alpha} \xi_{2 \mu}{ }^{\beta} \xi_{1}^{A} \\
\left(\hat{\mathcal{L}}_{\xi_{1}} \xi_{2}\right)_{\mu \nu}{ }^{\alpha}= & \left(L_{\xi_{1}} \xi_{2}\right)_{\mu \nu}^{\alpha}-3 \xi_{2}^{\rho} \partial_{[\rho} \xi_{1 \mu \nu]}^{\alpha} \\
& +2 F_{A \beta}{ }^{\alpha}\left(\xi_{2[\mu}{ }^{\beta} \partial_{\nu]} \xi_{1}^{A}-\xi_{2}^{A} \partial_{[\mu} \xi_{1 \nu]}^{\beta}-\xi_{[1}^{A} \xi_{2] \mu \nu}{ }^{\beta}+\xi_{1[\mu}{ }^{\beta} \xi_{2 \nu]}{ }^{A}\right)
\end{aligned}
$$

\subsection{The next step in the hierarchy $(133+8645)$ and so on...}

Recalling (3.18) we see that the last component in (3.20) is actually the result of a new projection due to the new intertwining tensor

$$
\begin{aligned}
F_{\mathcal{A}}{ }^{\alpha}\left[\left(\hat{\mathcal{L}}_{\xi_{1}} \xi_{2}\right)_{\mu \nu}{ }^{\mathcal{A}}=\left(L_{\xi_{1}} \xi_{2}\right)_{\mu \nu}{ }^{\mathcal{A}}-3 \xi_{2}^{\rho} \partial_{[\rho} \xi_{1 \mu \nu]} \mathcal{A}\right. & \\
& \left.+2 S^{\mathcal{A}} B \delta\left(\xi_{2[\mu}{ }^{\delta} \partial_{\nu]} \xi_{1}^{B}-\xi_{2}^{B} \partial_{[\mu} \xi_{1 \nu]}{ }^{\delta}-\xi_{[1}^{B} \xi_{2] \mu \nu}{ }^{\mathcal{B}} F_{\mathcal{B}}{ }^{\delta}-\xi_{2[\mu}{ }^{\delta} \xi_{1 \nu]}{ }^{\gamma} F_{\gamma}{ }^{B}\right)+\Gamma_{\mu \nu}{ }^{\mathcal{A}}\right]
\end{aligned}
$$

where we have introduced potential new contributions that vanish due to the projection

$$
\Gamma_{\mu \nu}^{\mathcal{A}} F_{\mathcal{A}}{ }^{\alpha}=0
$$

and defined $S^{\mathcal{A}}{ }_{B \delta}$ as a projector to the $\mathbf{9 1 2}$.

We can now proceed as in the previous subsection, and evaluate the failure of the closure for the unprojected components, setting for the moment $\Gamma_{\mu \nu} \mathcal{A}=0$. A long computation shows that

$$
\begin{aligned}
\left(\hat{\Delta}_{\xi_{2}} \hat{\mathcal{L}}_{\xi_{1}} \xi_{2}\right)_{\mu \nu} & \mathcal{A} \\
=F_{B C \alpha}{ }^{\mathcal{A}} & {\left[3 \xi _ { 2 } ^ { \rho } \left(\left(t_{\beta}\right)^{B C}\left(2 \xi_{1[\mu}{ }^{\alpha} \partial_{\nu} \xi_{3 \rho]}{ }^{\beta}+\left(\xi_{3[\rho}{ }^{\alpha} \xi_{1 \mu \nu]}{ }^{\mathcal{B}}-\xi_{1[\rho}{ }^{\alpha} \xi_{3 \mu \nu]}{ }^{\mathcal{B}}\right) F_{\mathcal{B}}{ }^{\beta}\right)\right.\right.} \\
& \left.-S_{\mathcal{B}}{ }^{C \alpha}\left(\xi_{1[\mu \nu}{ }^{\mathcal{B}} \partial_{\rho]} \xi_{3}^{B}+\xi_{1}^{B} \partial_{[\rho} \xi_{3 \mu \nu]}{ }^{\mathcal{B}}\right)\right)+\left(t_{\beta}\right)^{B C} \xi_{1}^{\rho} \xi_{3 \rho}{ }^{\alpha} \xi_{2 \mu \nu}{ }^{\mathcal{B}} F_{\mathcal{B}}{ }^{\beta} \\
& \left.+2 \xi_{2[\mu}{ }^{\alpha} \xi_{3 \nu]}{ }^{\gamma} F_{\gamma}{ }^{C} \xi_{1}^{B}-2 \xi_{2[\mu}{ }^{\alpha} \xi_{1 \nu]}{ }^{\gamma} F_{\gamma}{ }^{C} \xi_{3}^{B}+\left(2 \xi_{[1}^{B} \xi_{3] \mu \nu}{ }^{\mathcal{B}} \xi_{2}^{C}+\xi_{3}^{B} \xi_{1}^{C} \xi_{2 \mu \nu}{ }^{\mathcal{B}}\right) F_{\mathcal{B}}{ }^{\alpha}\right]
\end{aligned}
$$

As before, we have been able to factorize the new intertwining tensor

$$
F_{B C \alpha}{ }^{\mathcal{A}}=-2 S^{\mathcal{A}} C_{\beta}\left(t^{\beta}\right)_{B D} F_{\alpha}{ }^{D}+S^{\mathcal{A}}{ }_{D \alpha} F_{B C}{ }^{D}+2 S^{\mathcal{A}}{ }_{[B \mid \beta} F_{\mid C] \alpha}{ }^{\beta}
$$

We will collectively denote its indices $\mathbf{A}=B C \alpha$. As expected, this intertwining tensor is canceled through a projection with the previous one

$$
F_{\mathbf{A}}^{\mathcal{A}} F_{\mathcal{A}}^{\alpha}=0
$$


due to the quadratic constraints. It also satisfies the properties

$$
F_{B C}^{\alpha \mathcal{A}}=P_{(912) C}^{\alpha{ }^{D}}{ }_{\beta} F_{B D}^{\beta \mathcal{A}}, \quad F_{(B C) \alpha}{ }^{\mathcal{A}}=2\left(t^{\beta}\right)_{B C} S^{\mathcal{A}}{ }_{D[\beta} F_{\alpha]}^{D}
$$

The indices $C \alpha$ actually belong to the $\mathbf{9 1 2}$ and $\mathbf{A}=B C \alpha$ belongs to the $\mathbf{8 6 4 5}+\mathbf{1 3 3}$.

Now, following the route of the previous section, and inspired by (3.17), we propose

$$
\Gamma_{\mu \nu}^{\mathcal{A}}=\xi_{2}^{\rho} \xi_{1 \rho \mu \nu}{ }^{\mathcal{A}}+F_{A \mathcal{B}} \mathcal{A}^{\mathcal{B}} \xi_{2 \mu \nu}{ }^{\mathcal{B}} \xi_{1}^{A}
$$

where we have introduced new gauge parameters $\xi_{\rho \mu \nu}{ }^{\mathcal{A}}=F_{\mathbf{A}}{ }^{\mathcal{A}} \xi_{\rho \mu \nu} \mathbf{A}$. Another long computation shows that now full closure is achieved if

$$
\begin{aligned}
\left(\hat{\mathcal{L}}_{\xi_{1}} \xi_{2}\right)_{\rho \mu \nu}{ }^{\mathcal{A}}= & \left(L_{\xi_{1}} \xi_{2}\right)_{\rho \mu \nu}{ }^{\mathcal{A}}-4 \xi_{2}^{\sigma} \partial_{[\sigma} \xi_{1 \rho \mu \nu]} \mathcal{A} \\
& +F_{A \mathcal{B}} \mathcal{A}^{\mathcal{A}}\left[3\left(t_{\beta}\right)^{A C} S^{\mathcal{B}}{ }_{C \alpha}\left(2 \xi_{2[\mu}{ }^{\alpha} \partial_{\nu} \xi_{1 \rho]}{ }^{\beta}+\xi_{1[\rho}{ }^{\alpha} \xi_{2 \mu \nu]}{ }^{\beta}-\xi_{2[\rho}{ }^{\alpha} \xi_{1 \mu \nu]}{ }^{\beta}\right)\right. \\
& \left.+3 \xi_{2[\mu \nu}{ }^{\mathcal{B}} \partial_{\rho]} \xi_{1}^{A}+3 \xi_{2}^{A} \partial_{[\rho} \xi_{1 \mu \nu]}{ }^{\mathcal{B}}-2 \xi_{[2}^{A} \xi_{1] \rho \mu \nu} \mathcal{B}\right]
\end{aligned}
$$

Again, one could now extract the projection of the intertwining tensor from this expression, and add new terms to achieve closure, which will take the form

$$
\Gamma_{\rho \mu \nu}{ }^{\mathbf{A}}=\xi_{2}^{\sigma} \xi_{1 \sigma \rho \mu \nu} \mathbf{A}^{\mathbf{A}}-F_{A \mathbf{B}} \mathbf{A}_{\xi_{2 \rho \mu \nu}} \mathbf{B}_{1}^{A}
$$

with $F_{A \mathbf{B}}{ }^{\mathbf{A}}$ the next intertwining tensor. In principle one can repeat these steps over and over and build the so-called tensor hierarchy. Note however that a 4 -form gauge parameter is supposed to transform a 5-form, which cannot be present in 4-dimensions, and we will then stop here.

\subsection{The full generalized Lie derivative}

We have been able to construct, step by step, a generalized Lie derivative that incorporates the diffeomorphisms and gauge transformations of gauged maximal supergravity. Schematically, it takes the form

$$
\left(\hat{\mathcal{L}}_{\xi_{1}} \xi_{2}\right)^{\mathbb{A}}=\xi_{1}^{\mathbb{B}} \partial_{\mathbb{B}} \xi_{2}^{\mathbb{A}}-\xi_{2}^{\mathbb{B}} \partial_{\mathbb{B}} \xi_{1}^{\mathbb{A}}+W^{\mathbb{A}} \mathbb{B}_{\mathbb{D}} \partial_{\mathbb{C}} \xi_{1}^{\mathbb{D}} \xi_{2}^{\mathbb{B}}+F_{\mathbb{B C}}{ }^{\mathbb{A}} \xi_{1}^{\mathbb{B}} \xi_{2}^{\mathbb{C}}
$$

where we have collectively denoted the indices $\xi^{\mathbb{A}}=\left(\xi^{\mu}, \xi^{A}, \xi_{\mu}{ }^{\alpha}, \xi_{\mu \nu} \mathcal{A}, \xi_{\mu \nu \rho} \mathbf{A}, \ldots\right)$, and since we are working in 4 -dimensions we also have $\partial_{\mathbb{A}}=\left(\partial_{\mu}, 0, \ldots\right)$. The first three terms are un-gauged, and the $F_{\mathbb{B} C} \mathbb{A}^{\mathbb{A}}$ represents a collection of all the gaugings, mostly containing intertwiners. The $W^{\mathbb{A}} \mathbb{C}_{\mathbb{D}}$ tensor is formed by invariants of the symmetry group. Notice that the generalized Lie derivative is linear in derivatives. In particular, the gauged terms are not derived, but the gaugings are linear in internal derivatives. Also notice that only the gauge parameters that generate the transformation are derived. Its structure resembles the general structure of the gauged generalized Lie derivative of gauged DFT [62, 63, 65, 66]. The difference here is that the hierarchy of vectors requires a large extended (exceptional) tangent space, and this is due to the fact that the gaugings are not antisymmetric, and 
then there is a tower of intertwiners. The remarkable equivalence between the structure of both generalized Lie derivatives however suggests that this construction can be uplifted to a duality covariant construction in an extended space, as it is case for gauged DFT [62, 63]. Such a construction should be equipped with an un-gauged generalized Lie derivative in an extended space, and should reduce to this one upon generalized Scherk-Schwarz reduction. We will give later the first steps in this direction. Moreover, gauged DFT encodes halfmaximal gauged supergravities, and we will see later that this construction encodes the maximal gauged supergravities in 4-dimensions.

In components, the generalized Lie derivative (3.30) reads

$$
\begin{aligned}
& \left(\hat{\mathcal{L}}_{\xi_{1}} \xi_{2}\right)^{\mu}=\left(L_{\xi_{1}} \xi_{2}\right)^{\mu} \\
& \left(\hat{\mathcal{L}}_{\xi_{1}} \xi_{2}\right)^{A}=\left(L_{\xi_{1}} \xi_{2}\right)^{A}-\xi_{2}^{\rho} \partial_{\rho} \xi_{1}^{A}+F_{[B C]}^{A} \xi_{1}^{B} \xi_{2}^{C} \\
& +F_{(B C)}{ }^{A} \xi_{2}^{B} \xi_{1}^{C}+\xi_{2}^{\rho} \xi_{1 \rho}{ }^{A} \\
& \left(\hat{\mathcal{L}}_{\xi_{1}} \xi_{2}\right)_{\mu}{ }^{\alpha}=\left(L_{\xi_{1}} \xi_{2}\right)_{\mu}{ }^{\alpha}-2 \xi_{2}^{\rho} \partial_{[\rho} \xi_{1 \mu]}{ }^{\alpha}-S^{\alpha}{ }_{C B}\left(2 \xi_{2}^{C} \partial_{\mu} \xi_{1}^{B}+4 \xi_{[1}^{C} \xi_{2] \mu}{ }^{\beta} F_{\beta}{ }^{B}\right) \\
& -F_{A \beta}{ }^{\alpha} \xi_{2 \mu}{ }^{\beta} \xi_{1}^{A}+\xi_{2}^{\rho} \xi_{1 \rho \mu}{ }^{\alpha} \\
& \left(\hat{\mathcal{L}}_{\xi_{1}} \xi_{2}\right){ }_{\mu \nu}{ }^{\mathcal{A}}=\left(L_{\xi_{1}} \xi_{2}\right)_{\mu \nu}{ }^{\mathcal{A}}-3 \xi_{2}^{\rho} \partial_{[\rho} \xi_{1 \mu \nu]}{ }^{\mathcal{A}} \\
& -S^{\mathcal{A}}{ }_{C \beta}\left(2 \xi_{2}^{C} \partial_{[\mu} \xi_{1 \nu]}^{\beta}-2 \xi_{2[\mu}{ }^{\beta} \partial_{\nu]} \xi_{1}^{C}+2 \xi_{[1}^{C} \xi_{2] \mu \nu}{ }^{\mathcal{B}} F_{\mathcal{B}}{ }^{\beta}+2 \xi_{2[\mu}{ }^{\beta} \xi_{1 \nu]}{ }^{\gamma} F_{\gamma}^{C}\right) \\
& +F_{A \mathcal{B}} \mathcal{A}^{\mathcal{A}} \xi_{2 \mu \nu}{ }^{\mathcal{B}} \xi_{1}^{A}+\xi_{2}^{\rho} \xi_{1 \rho \mu \nu} \mathcal{A} \\
& \left(\hat{\mathcal{L}}_{\xi_{1}} \xi_{2}\right)_{\rho \mu \nu}{ }^{\mathbf{A}}=\left(L_{\xi_{1}} \xi_{2}\right)_{\rho \mu \nu}{ }^{\mathbf{A}}-4 \xi_{2}^{\sigma} \partial_{[\sigma} \xi_{1 \rho \mu \nu]} \mathbf{A} \\
& -S^{\mathbf{A}}{ }_{C \mathcal{B}}\left[3\left(t_{\beta}\right)^{C D} S^{\mathcal{B}}{ }_{D \alpha}\left(2 \xi_{2[\mu}{ }^{\alpha} \partial_{\nu} \xi_{1 \rho]}{ }^{\beta}+\left(\xi_{1[\rho}{ }^{\alpha} \xi_{2 \mu \nu]}{ }^{\mathcal{D}}-\xi_{2[\rho}{ }^{\alpha} \xi_{1 \mu \nu]}{ }^{\mathcal{D}}\right) F_{\mathcal{D}}{ }^{\beta}\right)\right. \\
& \left.+3 \xi_{2}^{C} \partial_{[\rho} \xi_{1 \mu \nu]}{ }^{\mathcal{B}}+3 \xi_{2[\mu \nu}{ }^{\mathcal{B}} \partial_{\rho]} \xi_{1}^{C}+2 \xi_{[1}^{C} \xi_{2] \rho \mu \nu}{ }^{\mathbf{B}} F_{\mathbf{B}}{ }^{\mathcal{B}}\right] \\
& -F_{A \mathbf{B}} \mathbf{A}_{\xi_{2 \rho \mu \nu}} \mathbf{B}_{\xi_{1}^{A}}^{A}+\xi_{2}^{\sigma} \xi_{1 \sigma \rho \mu \nu} \mathbf{A}
\end{aligned}
$$

Here, the $S$-tensors correspond to projectors to the different representations. One can rapidly identify a common structure in all the components. There is a hierarchy of intertwining tensors

$$
F_{\alpha}{ }^{A}, \quad F_{\mathcal{A}}{ }^{\alpha}, \quad F_{\mathbf{A}}^{\mathcal{A}}, \quad \cdots
$$

which have been defined in (3.11), (3.14) and (3.24). They are such that when a given component of the generalized Lie derivative is projected by its corresponding intertwining tensor, the sub algebra formed by it, together with the previous components, closes. The reason for this is that the contraction between successive intertwining tensors vanishes

$$
F_{\mathcal{A}}{ }^{\alpha} F_{\alpha}{ }^{A}=F_{\mathbf{A}}{ }^{\mathcal{A}} F_{\mathcal{A}}{ }^{\alpha}=\cdots=0
$$

and the failure of closure when the hierarchy is truncated to a given level, is proportional to the intertwiner of that level. 
Formally, one can extend this into an infinite hierarchy [39], but beyond the dimension of space-time the fields would vanish, and then the levels considered here are the physically relevant ones.

\subsection{Generalized bein}

We now have a closed form of the generalized Lie derivative. Due to partial projections by the intertwining tensors $F_{\alpha}{ }^{A}, F_{\mathcal{A}}{ }^{\alpha}, F_{\mathbf{A}}{ }^{\mathcal{A}}, \ldots$ one can achieve closure step by step. Here, we will propose a parameterization of the generalized frame or bein in terms of the bosonic degrees of freedom of gauged maximal supergravity, namely

- A 4-dimensional vielbein $e_{\bar{a}}{ }^{\mu}$, where the flat index $\bar{a}=1, \ldots, 4$ is acted on by the Lorentz group $\mathrm{SO}(1,3)$.

- 56 gauge vector fields $A_{\mu}{ }^{A}$.

- 70 scalars, parameterized by the coset matrix $\Phi_{\bar{A}}{ }^{A}$, where the flat index $\bar{A}=1, \ldots, 56$ is acted on by $\mathrm{SU}(8)$.

- 133 two-forms $B_{\mu \nu}{ }^{\alpha}$, which at the level of the Lagrangian are projected to 56 components due to the intertwining tensor $F_{\alpha}{ }^{A}$ in the formulation of [52].

- 912 three-forms $C_{\mu \nu \rho}{ }^{\mathcal{A}}$, with no dynamical degrees of freedom.

Moreover, enlarging the generalized frame to the full E-tangent space, we could add more and more fields in the tensor hierarchy, but we will stop here for simplicity.

Introducing indices $\mathbb{A}=\left({ }^{\mu},{ }^{A},{ }_{\mu}{ }^{\alpha},{ }_{\mu \nu} \mathcal{A}_{, \mu \nu \rho} \mathbf{A}, \ldots\right)$ and $\overline{\mathbb{A}}=\left({ }^{\bar{a}},{ }^{\bar{A}},{ }_{\bar{a}}{ }^{\bar{\alpha}}, \bar{a} \bar{b} \overline{\mathcal{A}}, \bar{a} \bar{b} \bar{c} \bar{A}, \ldots\right)$, we propose

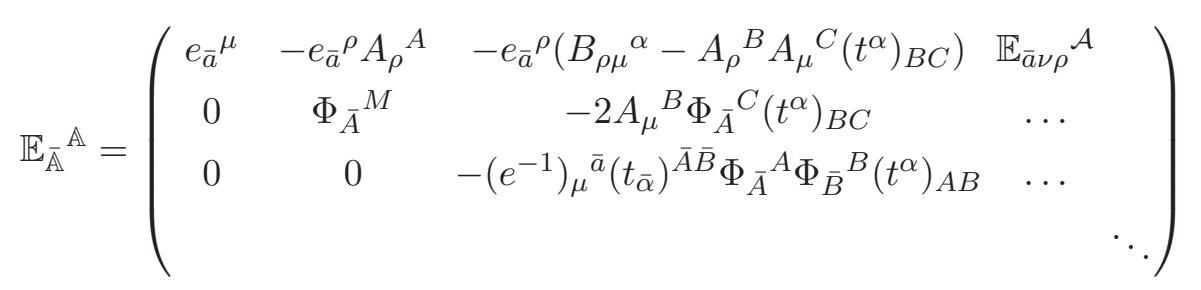

where the flat indices are naturally acted on by $H=\mathrm{SO}(1,3) \times \mathrm{SU}(8), \mathbb{E}_{\bar{a} \nu \rho} \mathcal{A}$ was defined in (2.16), and the remaining components would contain the higher $p$-forms.

Inserting this in the generalized Lie derivative, we obtain the gauge transformations of each component

$$
\begin{aligned}
\hat{\delta}_{\xi} e_{\bar{a}}{ }^{\mu}= & L_{\xi} e_{\bar{a}}{ }^{\mu} \\
\hat{\delta}_{\xi} A_{\mu}{ }^{A}= & L_{\xi} A_{\mu}{ }^{A}+\partial_{\mu} \xi^{A}+F_{B C}{ }^{A} \xi^{B} A_{\mu}{ }^{C}-\xi_{\mu}{ }^{A} \\
\hat{\delta}_{\xi} \Phi_{\bar{A}}{ }^{A}= & L_{\xi} \Phi_{\bar{A}}{ }^{A}+F_{B C}{ }^{A} \xi^{B} \Phi_{\bar{A}}{ }^{C} \\
\hat{\delta}_{\xi} B_{\mu \nu}{ }^{\alpha}= & L_{\xi} B_{\mu \nu}{ }^{\alpha}+2 \partial_{[\mu} \xi_{\nu]}{ }^{\alpha}-\xi_{\mu \nu}{ }^{\alpha}+2\left(t^{\alpha}\right)_{B C}\left(A_{[\mu}{ }^{B} \xi_{\nu]}{ }^{C}-A_{[\mu}{ }^{B} \partial_{\nu]} \xi^{C}\right) \\
& -F_{A \beta}{ }^{\alpha} \xi^{A} B_{\mu \nu}{ }^{\beta}-2\left(t^{\alpha}\right)_{B C} \xi^{B} F_{\beta}{ }^{C} B_{\mu \nu}{ }^{\beta}
\end{aligned}
$$


which faithfully reproduce those of gauged maximal supergravity in the formulation of [52]. Considering the remaining components would allow to make contact with [37].

Following the usual geometric constructions in extended geometries, building generalized connections and curvatures, one should be able to reproduce the bosonic sector of gauged maximal supergravity in [52] and even construct a democratic formulation containing the other $p$-forms. In fact, we will see in the next subsection that the so-called generalized fluxes encode all the covariant structures of the theory.

\subsection{Generalized fluxes}

We now define the so-called generalized fluxes

$$
\mathbb{F}_{\overline{\mathbb{A}} \overline{\mathbb{B}}} \overline{\overline{\mathbb{C}}}=\left(\hat{\mathcal{L}}_{\mathbb{E}_{\overline{\mathbb{A}}}} \mathbb{E}_{\overline{\mathbb{B}}}\right)^{\mathbb{C}}\left(\mathbb{E}^{-1}\right)_{\mathbb{C}} \overline{\mathbb{C}}
$$

Since they are defined through the generalized Lie derivative, they can only define quantities that are covariant with respect to the gauge transformations. Moreover, since the vectors involved in their definition are given by generalized beins, we expect them to correspond to covariant derivatives and curvatures. The simplest ones that depend on the degrees of freedom in (3.34) are

$$
\begin{aligned}
\mathbb{F}_{\bar{a} \bar{b}}{ }^{\bar{c}} & =2 e_{[\bar{a}}{ }^{\rho} \partial_{\rho} e_{\bar{b}]}^{\sigma} e_{\sigma}^{\bar{c}}=\omega_{[\bar{a} \bar{b}]}^{\bar{c}} \\
\mathbb{F}_{\bar{a} \bar{b}} \bar{C} & =-e_{\bar{a}}^{\mu} e_{\bar{b}}^{\nu}\left(\Phi^{-1}\right)_{C} \bar{C} \mathcal{F}_{\mu \nu}{ }^{C} \\
\mathbb{F}_{\bar{a} \bar{b} \bar{c}} \bar{\gamma} & =e_{\bar{a}}{ }^{\mu} e_{\bar{b}}^{\nu} e_{\bar{c}}{ }^{\rho}\left(t_{\alpha}\right)^{A B}\left(\Phi^{-1}\right)_{A}{ }^{\bar{A}}\left(\Phi^{-1}\right)_{B}{ }^{\bar{B}}\left(t^{\bar{\gamma}}\right)_{\bar{A} \bar{B}} \mathcal{H}_{\mu \nu \rho}{ }^{\alpha} \\
\mathbb{F}_{\bar{A} \bar{B} \bar{B}} \bar{C} & =\Phi_{\bar{A}}{ }^{A} \Phi_{\bar{B}}{ }^{B}\left(\Phi^{-1}\right)_{C}{ }^{\bar{C}} F_{A B}{ }^{C} \\
\mathbb{F}_{\bar{a} \bar{B} \bar{C}} \bar{C} & =-\mathbb{F}_{\bar{B} \bar{a}} \bar{C}=e_{\bar{a}}{ }^{\mu}\left(\Phi^{-1}\right)_{C}{ }^{\bar{C}} D_{\mu} \Phi_{\bar{B}}^{C}
\end{aligned}
$$

where

$$
\begin{aligned}
D_{\mu} \Phi_{\bar{B}}{ }^{C} & =\partial_{\mu} \Phi_{\bar{B}}{ }^{C}-F_{A B}{ }^{C} A_{\mu}{ }^{A} \Phi_{\bar{B}}{ }^{B} \\
\mathcal{F}_{\mu \nu}{ }^{C} & =2 \partial_{[\mu} A_{\nu]}{ }^{C}-F_{[A B]}{ }^{C} A_{\mu}{ }^{B} A_{\nu}{ }^{C}+B_{\mu \nu}{ }^{\alpha} F_{\alpha}{ }^{C} \\
\mathcal{H}_{\mu \nu \rho}{ }^{\alpha} & =3\left[\partial_{[\mu} B_{\nu \rho]}{ }^{\alpha}-C_{\mu \nu \rho}{ }^{\mathcal{A}} F_{\mathcal{A}}{ }^{\alpha}+2\left(t^{\alpha}\right)_{B C}\left(A_{[\mu}{ }^{B} \partial_{\nu} A_{\rho]}{ }^{C}+A_{[\mu}{ }^{B} B_{\nu \rho]}{ }^{\beta} F_{\beta}{ }^{C}\right.\right. \\
& \left.\left.+\frac{1}{3} F_{D E}{ }^{B} A_{[\mu}{ }^{D} A_{\nu}{ }^{E} A_{\rho]}{ }^{C}\right)\right]
\end{aligned}
$$

Here we can rapidly identify the antisymmetric part of the 4-dimensional spin connection, the curvatures of the 1-forms and 2-forms, the covariant derivatives of the scalars and the gaugings. All these correspond to covariant quantities. Note that to compute the curvature of the two-form, we need the bein component defied in (2.16). As before, one can further extend this analysis so as to make contact with the higher-level curvatures in [37].

In extended geometric constructions, the action (or generalized Ricci scalar) is quadratic in generalized fluxes (see for example [62, 63]). Then, the covariant generalized Ricci scalar constructed from these generalized fluxes will include a 4-dimensional 
Ricci scalar originated from the spin connection above, kinetic terms for the scalars originated from the covariant derivatives of scalars above, kinetic terms for the gauge fields originated from the field strengths above, and so on. In other words, the generalized fluxes we have found are precisely the covariant quantities that enter the action of gauged maximal supergravity. Furthermore, the other fluxes contained in this formulation would allow to build in a generalized geometrical fashion a democratic formulation of 4-dimensional maximal supergravity, like the one explored in [58].

\subsection{Generalized Bianchi Identities}

Given that the generalized Lie derivative transforms tensors into tensors, the closure conditions correspond to Bianchi Identities. We can then define

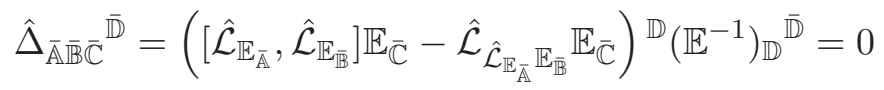

More explicitly this is

$$
\begin{aligned}
& \hat{\Delta}_{\overline{\mathbb{D}} \overline{\mathbb{A}} \overline{\mathbb{B}}} \overline{\bar{C}}=\hat{\Delta}_{\bar{E}_{\mathbb{D}}} \mathbb{F}_{\overline{\mathbb{A}} \overline{\mathbb{B}}} \overline{\mathbb{C}}
\end{aligned}
$$

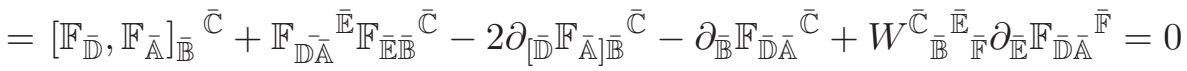

These generalized BI include those of the four dimensional Riemann tensor and that of the curvature for the two form (for simplicity we compute only the projection of the latter)

$$
\begin{aligned}
\hat{\Delta}_{\bar{d} \bar{a} \bar{b}}{ }^{\bar{c}} & =3 e_{\bar{a}}^{\mu} e_{\bar{d}}^{\nu} e_{\bar{b}}^{\rho}\left(e^{-1}\right)_{\sigma}{ }^{\bar{c}} R_{[\mu \nu \rho]}^{\sigma}=3\left(\partial_{[\bar{a}} \omega_{\overline{d b}]}^{\bar{c}}+\omega_{[\bar{a} \bar{d}} \bar{e}_{\bar{b}} \omega_{\bar{b}] \bar{e}}^{\bar{c}}\right) \\
\hat{\Delta}_{\bar{d} \bar{a} \bar{b}} \bar{C} & =e_{\bar{d}}^{\mu} e_{\bar{a}}^{\nu} e_{\bar{b}}{ }^{\rho}\left(\Phi^{-1}\right)_{M} \bar{C}\left(3 D_{[\mu} \mathcal{F}_{\nu \rho]}{ }^{M}-\mathcal{H}_{\mu \nu \rho}{ }^{M}\right)
\end{aligned}
$$

but more generally encode all other possible BI. For example, pursuing with these computations one should obtain all the BIs of [40].

\section{Generalized diffeomorphisms in extended geometry}

In this section we address the construction of a full generalized Lie derivative in the extended mega-space-time associated to the U-duality groups in M-theory. Using the particular $E_{7(7)}$ case analyzed in the previous section as a guide line, here we will start general and do not specify any particular duality group. Beginning with the canonical extension of a Lie derivative under a Kaluza-Klein decomposition to the U-duality case, we compute the closure and its failure. This allows to explore the extensions that form a closed algebra, the appearance of the tensor hierarchy and the role of intertwiners.

\subsection{The canonical extension and the failure of closure}

We could start with a Lie derivative in higher-dimensions (60-in the $E_{7(7)}$ case) and split indices in "external" and "internal" components, namely $\left(\hat{V}^{\mu}(x, Y), \hat{V}^{M}(x, Y)\right)$. Now the vectors depend on "external" $x$ and "internal" $Y$ coordinates, and to make a difference with the vectors of the previous section, here we put a hat on them. Although we name the indices as internal and external, let us emphasize that this just corresponds to an index 
splitting, and here we are not assuming any compactification-type ansatz. In components it takes the form

$$
\begin{aligned}
\left(\hat{\mathcal{L}}_{\hat{\xi}} \hat{V}\right)^{\mu} & =\left(L_{\hat{\xi}} \hat{V}\right)^{\mu}+\left(\mathcal{L}_{\hat{\xi}} \hat{V}\right)^{\mu}-\left(\mathcal{L}_{\hat{V}} \hat{\xi}\right)^{\mu} \\
\left(\hat{\mathcal{L}}_{\hat{\xi}} \hat{V}\right)^{M} & =L_{\hat{\xi}} \hat{V}^{M}-\hat{V}^{\rho} \partial_{\rho} \hat{\xi}^{M}+\left(\mathcal{L}_{\hat{\xi}} \hat{V}\right)^{M}
\end{aligned}
$$

While in a conventional Kaluza-Klein splitting $\mathcal{L}_{\hat{\xi}} \hat{V}$ would correspond to the usual internal Lie derivative, here we promote it to the internal extended generalized Lie derivative

$$
\begin{aligned}
\left(\mathcal{L}_{\hat{\xi}} \hat{V}\right)^{\mu} & =\hat{\xi}^{P} \partial_{P} \hat{V}^{\mu}+\lambda \partial_{P} \hat{\xi}^{P} \hat{V}^{\mu} \\
\left(\mathcal{L}_{\hat{\xi}} \hat{V}\right)^{M} & =\hat{\xi}^{P} \partial_{P} \hat{V}^{M}-\hat{V}^{P} \partial_{P} \hat{\xi}^{M}+Y^{M}{ }_{N}{ }^{P}{ }_{Q} \partial_{P} \hat{\xi}^{Q} \hat{V}^{N}
\end{aligned}
$$

This internal part was introduced in [20-22], and here we are allowing the external part to carry a weight $\lambda$. By now we will remain general and do not select a particular $Y$-tensor, later we will specialize to different cases.

We know that when this extended generalized Lie derivative is restricted purely to the internal sector, the closure is achieved up to the so-called closure constraints [44]. In particular, under a SS compactification, these reproduce the quadratic constraints for the gaugings in gauged maximal supergravity. On the other hand, the purely external sector closes automatically, since it is governed by the 4-dimensional Lie derivative of Riemannian geometry. The combined case is however more tricky, and we can already state that it will not close. In fact, under a generalized Scherk-Schwarz dimensional reduction it reduces to (3.1), which as we saw required an extension.

Let us however proceed with the computation of closure, to see what the failure looks like in this more general case. After some algebra, we find the following result for the external components

$$
\begin{aligned}
\left(\hat{\Delta}_{\hat{\xi}_{1}} \hat{\mathcal{L}}_{\hat{\xi}_{2}} \hat{V}\right)^{\mu}= & Y^{P}{ }_{M}{ }^{Q}{ }_{N}\left[\partial_{Q} \hat{\xi}_{1}^{N} \hat{\xi}_{2}^{M} \partial_{P} \hat{V}^{\mu}+\lambda \partial_{P}\left(\partial_{Q} \hat{\xi}_{1}^{N} \hat{\xi}_{2}^{M}\right) \hat{V}^{\mu}\right. \\
& \left.+2\left(\partial_{P} \hat{\xi}_{[1}^{\mu} \partial_{Q} \hat{\xi}_{2]}^{N} \hat{V}^{M}+\lambda \partial_{P}\left(\partial_{Q} \hat{\xi}_{[2}^{N} \hat{V}^{M}\right) \hat{\xi}_{1]}^{\mu}\right)\right] \\
+\lambda[ & \left(\partial_{P} \hat{\xi}_{1}^{P}\right)\left(L_{\hat{\xi}_{2}} \hat{V}\right)^{\mu}+\left(\partial_{P} \hat{V}^{P}\right)\left(L_{\hat{\xi}_{1}} \hat{\xi}_{2}\right)^{\mu}+\left(\partial_{P} \hat{\xi}_{2}^{P}\right)\left(L_{\hat{V}} \hat{\xi}_{1}\right)^{\mu} \\
& +\hat{V}^{\mu}\left(\partial_{P} \hat{\xi}_{1}^{\rho} \partial_{\rho} \hat{\xi}_{2}^{P}-\partial_{P} \hat{\xi}_{2}^{\rho} \partial_{\rho} \hat{\xi}_{1}^{P}\right)+\hat{\xi}_{2}^{\mu}\left(\partial_{P} \hat{V}^{\rho} \partial_{\rho} \hat{\xi}_{1}^{P}-\partial_{P} \hat{\xi}_{1}^{\rho} \partial_{\rho} \hat{V}^{P}\right) \\
& \left.+\hat{\xi}_{1}^{\mu}\left(\partial_{P} \hat{\xi}_{2}^{\rho} \partial_{\rho} \hat{V}^{P}-\partial_{P} \hat{V}^{\rho} \partial_{\rho} \hat{\xi}_{2}^{P}\right)\right]
\end{aligned}
$$

and the corresponding result for the internal components

$$
\begin{aligned}
\left(\hat{\Delta}_{\hat{\xi}_{1}} \hat{\mathcal{L}}_{\hat{\xi}_{2}} \hat{V}\right)^{M}= & 2 Y^{M}{ }_{N}{ }^{P}{ }_{Q} \hat{V}^{Q} \partial_{P} \hat{\xi}_{[1}^{\rho} \partial_{\rho} \hat{\xi}_{2]}^{N}-\hat{V}^{\rho}\left[\mathcal{L}_{\partial_{\rho} \hat{\xi}_{1}} \hat{\xi}_{2}+\mathcal{L}_{\hat{\xi}_{2}} \partial_{\rho} \hat{\xi}_{1}\right] M \\
- & -\left[\left(\left[\mathcal{L}_{\hat{\xi}_{1}}, \mathcal{L}_{\hat{\xi}_{2}}\right]-\mathcal{L}_{\mathcal{L}_{\hat{\xi}_{1}} \hat{\xi}_{2}}\right) \hat{V}\right]^{M} \\
& +\lambda\left[\left(\partial_{P} \hat{\xi}_{1}^{P}\right)\left(\hat{\xi}_{2}^{\rho} \partial_{\rho} \hat{V}^{M}-\hat{V}^{\rho} \partial_{\rho} \hat{\xi}_{2}^{M}\right)+\left(\partial_{P} \hat{\xi}_{2}^{P}\right)\left(\hat{V}^{\rho} \partial_{\rho} \hat{\xi}_{1}^{M}-\hat{\xi}_{1}^{\rho} \partial_{\rho} \hat{V}^{M}\right)\right. \\
& \left.\quad+\left(\partial_{P} \hat{V}^{P}\right)\left(\hat{\xi}_{1}^{\rho} \partial_{\rho} \hat{\xi}_{2}^{M}-\hat{\xi}_{2}^{\rho} \partial_{\rho} \hat{\xi}_{1}^{M}\right)\right]
\end{aligned}
$$


Let us now analyze these results. We have split the external components (4.3) in two blocks. The first one vanishes by imposing the internal closure constraints, but the second one does not. However, the latter is proportional to $\lambda$, and then we can guarantee closure for the external components provided we take

$$
\lambda=0
$$

We will assume this from now on. Then, in the internal components (4.4) the last block vanishes. The second line in (4.4) corresponds to the closure of the internal sector. It was shown in [44] that this vanishes either by imposing the section condition (2.2), or the internal closure constraints implemented in Scherk-Schwarz reductions (see for example (3.4)). The first line is however problematic, it does not vanish due to the section condition, nor (as we saw) upon imposing the quadratic constraints in Scherk-Schwarz reductions. In fact, under such a compactification ansatz while the first term compactifies to zero (we assume that under a compactification the external components depend on external coordinates only), the second term compactifies to an intertwining-dependent term, like that of (3.4).

Then, for weight zero external components (4.5) and restricted vectors (either due to the section condition or due to the more relaxed internal closure constraints) the generalized Lie derivative (4.1) fails to close up to the following terms

$$
\begin{aligned}
\left(\hat{\Delta}_{\hat{\xi}_{1}} \hat{\mathcal{L}}_{\hat{\xi}_{2}} \hat{V}\right)^{\mu}= & 0 \\
\left(\hat{\Delta}_{\hat{\xi}_{1}} \hat{\mathcal{L}}_{\hat{\xi}_{2}} \hat{V}\right)^{M}= & 2 Y^{M}{ }_{P}{ }^{N}{ }_{Q} \partial_{N} \hat{\xi}_{[1}^{\mu} \partial_{\mu} \hat{\xi}_{2]}^{Q} \hat{V}^{P}-\hat{V}^{\mu} Y^{M}{ }_{P}{ }^{N}{ }_{Q} \partial_{N}\left(\partial_{\mu} \hat{\xi}_{1}^{Q} \hat{\xi}_{2}^{P}\right) \\
& +2 \hat{V}^{\mu} Y^{M}{ }_{[P}{ }^{N}{ }_{Q]} \partial_{\mu} \hat{\xi}_{1}^{Q} \partial_{N} \hat{\xi}_{2}^{P}
\end{aligned}
$$

and then implies, as expected, that the extended generalized Lie derivative must be completed.

\subsection{Including the tensor hierarchy}

The structure of the failure (4.6) suggests the way in which the generalized Lie derivative must be completed. In particular, the structure of the first two terms suggest introducing the underlined terms

$$
\begin{aligned}
& \left(\hat{\mathcal{L}}_{\hat{\xi}_{1}} \hat{\xi}_{2}\right)^{\mu}=\left(L_{\hat{\xi}_{1}} \hat{\xi}_{2}\right)^{\mu}+\hat{\xi}_{1}^{P} \partial_{P} \hat{\xi}_{2}^{\mu}-\hat{\xi}_{2}^{P} \partial_{P} \hat{\xi}_{1}^{\mu} \\
& \left(\hat{\mathcal{L}}_{\hat{\xi}_{1}} \hat{\xi}_{2}\right)^{M}=L_{\hat{\xi}_{1}} \hat{\xi}_{2}^{M}-\hat{\xi}_{2}^{\rho} \partial_{\rho} \hat{\xi}_{1}^{M}+\left(\mathcal{L}_{\hat{\xi}_{1}} \hat{\xi}_{2}\right)^{M} \\
& +\frac{1}{2} \frac{Y_{P}^{M}{ }_{P}{ }_{Q} \partial_{N} \hat{\xi}_{1 \rho}{ }^{P Q} \hat{\xi}_{2}^{\rho}}{2}+\frac{1}{2} \frac{Y^{M}{ }_{P}{ }^{N}{ }_{Q} \hat{\xi}_{2 \rho} P Q}{P Q} \partial_{N} \hat{\xi}_{1}^{\rho}
\end{aligned}
$$

We have included new parameters $\hat{\xi}_{\rho}^{P Q}$, but note that in both terms they are projected by the $Y$-tensor. This tensor selects the relevant representations in the different duality groups. Let us anticipate the result: this extension works for all $E_{n+1(n+1)}, n<6$ but it fails for $E_{7(7)}$. 
If one computes closure with this generalized Lie derivative, without assuming any particular form for the invariant tensor $Y$, the result for the internal sector reads

$$
\begin{aligned}
\left(\hat{\Delta}_{\hat{\xi}_{1}} \hat{\mathcal{L}}_{\hat{\xi}_{2}} \hat{V}\right)^{\mu}=\frac{1}{2} Y_{R}^{P}{ }_{R}^{T}{ }_{S}[ & \left(2 \partial_{T} \hat{\xi}_{1}^{S} \hat{\xi}_{2}^{R}+\hat{\xi}_{2}^{\rho} \partial_{T} \hat{\xi}_{1 \rho}^{R S}+\hat{\xi}_{2 \rho}{ }^{R S} \partial_{T} \hat{\xi}_{1}^{\rho}\right) \partial_{P} \hat{V}^{\mu} \\
& -\left(2 \partial_{T} \hat{\xi}_{1}^{S} \hat{V}^{R}+\hat{V}^{\rho} \partial_{T} \hat{\xi}_{1 \rho}{ }^{R S}+\hat{V}_{\rho}^{R S} \partial_{T} \hat{\xi}_{1}^{\rho}\right) \partial_{P} \hat{\xi}_{2}^{\mu} \\
& \left.-\left(2 \partial_{T} \hat{\xi}_{2}^{S} \hat{V}^{R}+\hat{V}^{\rho} \partial_{T} \hat{\xi}_{2 \rho}{ }^{R S}+\hat{V}_{\rho}^{R S} \partial_{T} \hat{\xi}_{2}^{\rho}\right) \partial_{P} \hat{\xi}_{1}^{\mu}\right]
\end{aligned}
$$

Every single term here is of the form $Y^{P}{ }^{T}{ }_{S} \partial_{P} \otimes \partial_{T}$, so this vanishes under the section condition (2.2), but more generally these correspond to closure constraints that compactify to zero.

Moving to the internal sector, after a long computation the closure of the algebra can be taken to the form

$$
\begin{aligned}
& \left(\hat{\Delta}_{\hat{\xi}_{1}} \hat{\mathcal{L}}_{\hat{\xi}_{2}} \hat{V}\right)^{M}=\frac{1}{2} \hat{V}^{\mu} Y^{M}{ }_{P}{ }^{N}{ }_{Q} \partial_{N}\left[\left(\hat{\delta}_{\hat{\xi}_{1}} \hat{\xi}_{2}\right)_{\mu}{ }^{P Q}-L_{\hat{\xi}_{1}} \hat{\xi}_{2 \mu}{ }^{P Q}+2 \hat{\xi}_{2}^{\rho} \partial_{[\rho} \hat{\xi}_{1 \mu]} P Q-2 \partial_{\mu} \hat{\xi}_{1}^{Q} \hat{\xi}_{2}^{P}\right. \\
& \left.+2 Y_{R}^{P}{ }_{R}^{T}{ }_{S} \hat{\xi}_{[2}^{Q} \partial_{T} \hat{\xi}_{1] \mu}^{R S}+\partial_{T}\left(Y^{P}{ }_{R}^{T}{ }_{S} \hat{\xi}_{1}^{Q} \hat{\xi}_{2 \mu}{ }^{R S}-\hat{\xi}_{1}^{T} \hat{\xi}_{2 \mu}{ }^{P Q}\right)\right] \\
& -\partial_{N} \hat{\xi}_{[1}^{\mu} Y^{M}{ }_{P}{ }^{N}\left[\left(\hat{\delta}_{\left.\hat{\xi}_{2}\right]} \hat{V}\right)_{\mu}{ }^{P Q}-L_{\hat{\xi}_{2]}} \hat{V}_{\mu}^{P Q}+2 \hat{V}^{\rho} \partial_{[\rho} \hat{\xi}_{2] \mu]}^{P Q}-2 \partial_{\mu} \hat{\xi}_{2]}^{Q} \hat{V}^{P}\right. \\
& \left.+Y^{P}{ }_{R}^{T}{ }_{S}\left(\hat{V}^{Q} \partial_{T} \hat{\xi}_{2] \mu}{ }^{R S}-\hat{\xi}_{2]}^{Q} \partial_{T} \hat{V}_{\mu}^{R S}\right)+\partial_{T}\left(Y^{P}{ }_{R}^{T}{ }_{S} \hat{\xi}_{2]}^{Q} \hat{V}_{\mu}{ }^{R S}-\hat{\xi}_{2]}^{T} \hat{V}_{\mu}{ }^{P Q}\right)\right] \\
& +Y^{M}{ }_{\left[P^{N}\right.}{ }_{Q]}\left[\hat{V}^{\mu} Y^{P}{ }_{R}^{T}{ }_{S}\left(2 \partial_{N} \partial_{T} \hat{\xi}_{[2 \mu}{ }^{R S} \hat{\xi}_{1]}^{Q}-\partial_{N} \partial_{T}\left(\hat{\xi}_{1}^{Q} \hat{\xi}_{2 \mu}{ }^{R S}\right)\right)\right. \\
& \left.+2 Y_{R}^{P}{ }_{R}^{T}\left(\hat{V}^{Q} \partial_{T} \hat{\xi}_{[2 \rho}^{R S} \partial_{N} \hat{\xi}_{1]}^{\rho}+2 \hat{V}_{\rho}^{R S} \partial_{(N} \hat{\xi}_{[2}^{Q} \partial_{T)} \hat{\xi}_{1]}^{\rho}\right)+2 \hat{V}^{\mu} \partial_{\mu} \hat{\xi}_{1}^{Q} \partial_{N} \hat{\xi}_{2}^{P}\right] \\
& +\hat{\Delta}_{(S C)}^{M}
\end{aligned}
$$

where we have collected all the terms that would vanish under the section condition in the last term

$$
\begin{aligned}
& \hat{\Delta}_{(S C)}^{M}=\frac{1}{2}\left[Y_{Q}^{M}{ }_{Q}^{(N \mid}{ }_{P} Y^{P}{ }_{R}^{\mid T)}{ }_{S}-Y^{M}{ }_{R}{ }^{(N}{ }_{S} \delta_{Q}^{T)}\right] \\
& \left(2 \hat{V}^{Q} \partial_{T} \hat{\xi}_{2 \rho}^{R S} \partial_{N} \hat{\xi}_{1}^{\rho}+\hat{V}^{Q} \partial_{T} \partial_{N} \hat{\xi}_{1}^{\rho} \hat{\xi}_{2 \rho}^{R S}+4 \partial_{N} \hat{\xi}_{[2}^{Q} \partial_{T} \hat{\xi}_{1]}^{\rho} \hat{V}_{\rho}^{R S}\right. \\
& \left.+\hat{\xi}_{2}^{\rho} \hat{V}^{Q} \partial_{T} \partial_{N} \hat{\xi}_{1 \rho}^{R S}-\hat{V}^{\rho} \hat{\xi}_{[2}^{Q} \partial_{T} \partial_{N} \hat{\xi}_{1] \rho}^{R S}-\hat{V}^{\rho} \partial_{T} \partial_{N}\left(\hat{\xi}_{1}^{Q} \hat{\xi}_{2 \rho}^{R S}\right)\right) \\
& +\frac{1}{2} Y^{P}{ }_{R}^{T}{ }_{S}\left(\left(\hat{\xi}_{2}^{\rho} \partial_{T} \hat{\xi}_{1 \rho}^{R S}+\hat{\xi}_{2 \rho}{ }^{R S} \partial_{T} \hat{\xi}_{1}^{\rho}\right) \partial_{P} \hat{V}^{M}+2 \hat{V}_{\rho}^{R S} \partial_{T} \hat{\xi}_{[2}^{\rho} \partial_{P} \hat{\xi}_{1]}^{M}\right) \\
& +\left(\hat{\Delta}_{\hat{\xi}_{1}} \hat{\mathcal{L}}_{\hat{\xi}_{2}} \hat{V}\right) \stackrel{M}{(i)}
\end{aligned}
$$

The last term denoted by an $(i)$ contains the closure conditions of the internal sector, discussed in [44].

We will now discuss this in detail for specific duality groups.

\subsubsection{The T-duality case: double field theory}

When the duality group is $\mathrm{O}(n, n)$, namely T-duality, the $Y$-tensor is given by

$$
Y_{P}^{M}{ }^{N}=\eta^{M N} \eta_{P Q}
$$


where $\eta^{M N}$ is the symmetric duality invariant metric. In this case, the generalized Lie derivative (4.7) reduces to

$$
\begin{aligned}
\left(\hat{\mathcal{L}}_{\hat{\xi}_{1}} \hat{\xi}_{2}\right)^{\mu} & =\left(L_{\hat{\xi}_{1}} \hat{\xi}_{2}\right)^{\mu}+\hat{\xi}_{1}^{P} \partial_{P} \hat{\xi}_{2}^{\mu}-\hat{\xi}_{2}^{P} \partial_{P} \hat{\xi}_{1}^{\mu} \\
\left(\hat{\mathcal{L}}_{\hat{\xi}_{1}} \hat{\xi}_{2}\right)^{M} & =L_{\hat{\xi}_{1}} \hat{\xi}_{2}^{M}-\hat{\xi}_{2}^{\rho} \partial_{\rho} \hat{\xi}_{1}^{M}+\left(\mathcal{L}_{\hat{\xi}_{1}} \hat{\xi}_{2}\right)^{M}+\hat{\xi}_{2}^{\rho} \partial^{M} \hat{\xi}_{1 \rho}+\hat{\xi}_{2 \rho} \partial^{M} \hat{\xi}_{1}^{\rho}
\end{aligned}
$$

Here we have defined ${ }^{5}$

$$
\hat{\xi}_{\mu}=\frac{1}{2} \eta_{P Q} \hat{\xi}_{\mu}^{P Q}
$$

since the $Y$-tensor selects its pure trace, and then leaves a unique 1-form gauge parameter, and

$$
\left(\mathcal{L}_{\hat{\xi}_{1}} \hat{\xi}_{2}\right)^{M}=\hat{\xi}_{1}^{P} \partial_{P} \hat{\xi}_{2}^{M}-\hat{\xi}_{2}^{P} \partial_{P} \hat{\xi}_{1}^{M}+\partial^{M} \hat{\xi}_{1 P} \hat{\xi}_{2}^{P}
$$

which is now the usual internal generalized Lie derivative of DFT. In (4.9) all the last terms cancel for this $Y$-tensor, and the only terms left (up to contributions that would vanish due to the section condition) are the first four lines

$$
\begin{aligned}
&\left(\hat{\Delta}_{\hat{\xi}_{1}} \hat{\mathcal{L}}_{\hat{\xi}_{2}} \hat{V}\right)^{\mu}= 0 \\
&\left(\hat{\Delta}_{\hat{\xi}_{1}} \hat{\mathcal{L}}_{\hat{\xi}_{2}} \hat{V}\right)^{M}= \hat{V}^{\mu} \partial^{M}\left[\left(\hat{\delta}_{\hat{\xi}_{1}} \hat{\xi}_{2}\right)_{\mu}-\left(L_{\hat{\xi}_{1}} \hat{\xi}_{2}\right)_{\mu}+2 \hat{\xi}_{2}^{\rho} \partial_{[\rho} \hat{\xi}_{1 \mu]}\right. \\
&\left.-\partial_{\mu} \hat{\xi}_{1 P} \hat{\xi}_{2}^{P}+\hat{\xi}_{2}^{P} \partial_{P} \hat{\xi}_{1 \mu}-\hat{\xi}_{1}^{P} \partial_{P} \hat{\xi}_{2 \mu}\right] \\
& \quad-2 \partial^{M} \hat{\xi}_{2}^{\mu}\left[\left(\hat{\delta}_{\hat{\xi}_{1}} \hat{V}\right)_{\mu}-\left(L_{\hat{\xi}_{1}} \hat{V}\right)_{\mu}+2 \hat{V}^{\rho} \partial_{[\rho} \hat{\xi}_{1 \mu]}\right. \\
&\left.-\partial_{\mu} \hat{\xi}_{1 P} \hat{V}^{P}+\hat{V}^{P} \partial_{P} \hat{\xi}_{1 \mu}-\hat{\xi}_{1}^{P} \partial_{P} \hat{V}_{\mu}\right]+(1 \leftrightarrow 2)
\end{aligned}
$$

Setting this to zero selects the proper transformation rule for the 1-form leading to the generalized Lie derivative in DFT

$$
\begin{aligned}
\left(\hat{\mathcal{L}}_{\hat{\xi}_{1}} \hat{\xi}_{2}\right)^{\mu} & =\left(L_{\hat{\xi}_{1}} \hat{\xi}_{2}\right)^{\mu}+\hat{\xi}_{1}^{P} \partial_{P} \hat{\xi}_{2}^{\mu}-\hat{\xi}_{2}^{P} \partial_{P} \hat{\xi}_{1}^{\mu} \\
\left(\hat{\mathcal{L}}_{\hat{\xi}_{1}} \hat{\xi}_{2}\right)^{M} & =L_{\hat{\xi}_{1}} \hat{\xi}_{2}^{M}-\hat{\xi}_{2}^{\rho} \partial_{\rho} \hat{\xi}_{1}^{M}+\left(\mathcal{L}_{\hat{\xi}_{1}} \hat{\xi}_{2}\right)^{M}+\hat{\xi}_{2}^{\rho} \partial^{M} \hat{\xi}_{1 \rho}+\hat{\xi}_{2 \rho} \partial^{M} \hat{\xi}_{1}^{\rho} \\
\left(\hat{\mathcal{L}}_{\hat{\xi}_{1}} \hat{\xi}_{2}\right)_{\mu} & =\left(L_{\hat{\xi}_{1}} \hat{\xi}_{2}\right)_{\mu}-2 \hat{\xi}_{2}^{\rho} \partial_{[\rho} \hat{\xi}_{1 \mu]}+\partial_{\mu} \hat{\xi}_{1 P} \hat{\xi}_{2}^{P}-\hat{\xi}_{2}^{P} \partial_{P} \hat{\xi}_{1 \mu}+\hat{\xi}_{1}^{P} \partial_{P} \hat{\xi}_{2 \mu}
\end{aligned}
$$

This is the full generalized Lie derivative because in this case the last component of the closure conditions vanishes up to closure constraints

$$
\left(\hat{\Delta}_{\hat{\xi}_{1}} \hat{\mathcal{L}}_{\hat{\xi}_{2}} \hat{V}\right)_{\mu}=0
$$

Then, this is the end of the story here, only one additional one-form has to be included in order to achieve closure. This extra 1-form gauge parameter implies that there is only one 2 -form field in DFT, which is none but the Kalb-Ramond field $B_{\mu \nu}$. Interestingly, since in this case the number of 1 -forms and vectors coincides, all the gauge parameters can be

\footnotetext{
${ }^{5}$ This parameter should not be confused with the 4-dimensional vector $\hat{\xi}^{\mu}$, they correspond to completely different gauge parameters (the latter corresponds to 4-dimensional diffeomorphisms, while (4.14) to gauge transformations of $B_{\mu \nu}$, as we will see shortly).
} 
grouped into the bigger duality group $\mathrm{O}(d+n, d+n)$ of DFT, and the generalized Lie derivative can be condensed as

$$
\left(\hat{\mathcal{L}}_{\hat{\xi}_{1}} \hat{\xi}_{2}\right){ }^{\mathbb{M}}=\hat{\xi}_{1}^{\mathbb{P}} \partial_{\mathbb{P}} \hat{\xi}_{2}^{\mathbb{M}}-\hat{\xi}_{2}^{\mathbb{P}} \partial_{\mathbb{P}} \hat{\xi}_{1}^{\mathbb{M}}+Y^{\mathbb{M}} \mathbb{P}_{\mathbb{Q}}^{\mathbb{N}} \partial_{\mathbb{N}} \hat{\xi}_{1}^{\mathbb{Q}} \hat{\xi}_{2}^{\mathbb{P}}
$$

where we have noted $\hat{\xi}^{\mathbb{M}}=\left(\xi^{\mu}, \xi^{M}, \xi_{\mu}\right)$ and $\partial_{\mathbb{M}}=\left(\partial_{\mu}, \partial_{M}, 0\right)$, and $Y^{\mathbb{M}} \mathbb{P}_{\mathbb{Q}}=\eta^{\mathbb{M}} \eta_{\mathbb{P Q}}$, with $\eta^{\mathbb{M N}}$ the invariant metric of $\mathrm{O}(d+n, d+n)$. In DFT, one can incorporate derivatives with respect to the form directions $\partial^{\mu}$, and constrain or completely eliminate their dependence by imposing an external $\mathrm{O}(d, d)$ section condition. We expect that this is also the case in the general case of $U$-duality groups, although in this paper we are setting them to zero explicitly.

\subsubsection{The $E_{7(7)}$ case}

The above analysis fails to work for $E_{7(7)}$. The problem can already be tracked back to equation (4.6). The reason is that the last term, while vanishing in the cases of $\mathrm{O}(n, n)$ and $E_{n+1(n+1)}, n<6$, does not vanish when the $Y$-tensor is that of $E_{7(7)}$ because it contains an antisymmetric piece

$$
Y^{M}{ }_{P}^{N}{ }_{Q}=-12 P^{M N}(P Q)+\underline{\frac{1}{2} \omega^{M N} \omega_{[P Q]}}
$$

While the two extra terms in (4.7) cancel the failure of the first two terms in (4.6), there is no obvious covariant completion to the generalized Lie derivative that would cancel the last term in (4.6).

In close relation to this fact, although we have been able to close the algebra in the compactified case, by adding the extra term in (3.5), there is no obvious covariant uplift for this contribution, such that it compactifies to the form (3.8), namely, the new gauge parameter contracted with the intertwining tensor. To be more specific, let us note that the intertwining tensor $F_{(A B)}{ }^{C}$ can be cast as follows in terms of the twist matrix

$$
F_{(A B)}{ }^{C}=Y^{M}{ }_{P}{ }^{N}{ }_{Q} \partial_{N} U_{(A}{ }^{Q} U_{B)}{ }^{P}\left(U^{-1}\right)_{M}{ }^{C}
$$

Then, starting from the last term in the third line of (3.31), it should uplift to

$$
\xi_{\mu}{ }^{A B} F_{(A B)}{ }^{C} U_{C}{ }^{M}=\frac{1}{2} Y^{M}{ }_{P}{ }^{N}{ }_{Q} \partial_{N} \hat{\xi}_{\mu}^{P Q}+Y^{M}{ }_{[P}{ }^{N}{ }_{Q]} \partial_{N} U_{A}{ }^{Q} U_{B}{ }^{P} \xi_{\mu}{ }^{A B}
$$

where we assume that the Scherk-Schwarz ansatz is of the form $\hat{\xi}_{\mu}^{P Q}=U_{A}{ }^{P} U_{B}{ }^{Q} \xi_{\mu}{ }^{A B}$. Clearly the uplift should not be $U$-dependent, and then we see again that the failure to uplift the intertwining term in the reduced generalized Lie derivative is proportional to $Y^{M}{ }_{\left[P^{N}\right.}{ }_{Q]}$. Then, the result is that without adding a new contribution to (4.7) or supplementing it with some additional constraints, the closure fails to work up to terms proportional to the antisymmetric part of the $Y$-tensor (4.9).

Already in [46] there were indications that the $E_{7(7)}$ case is special in this respect: while the divergence of the 1-form gauge parameters is covariant (the connection contributions 
vanish) for $E_{n+1(n+1)}$ with $n<6$, this is not the case of $E_{7(7)}$. More concretely, for $n<6$ one has

$$
Y^{M}{ }_{P}^{N}{ }_{Q} \nabla_{N} \xi_{\mu}{ }^{P Q}=Y^{M}{ }_{P}{ }_{Q} \partial_{N} \xi_{\mu}{ }^{P Q}
$$

and then this expression is connection free. Then, one possibility is that the extracontributions in (4.9) should be actually defined in terms of a covariant derivative. Another possibility is implementing a field section condition. As we will see in the next section, when filling the fundamental $\mathbf{5 6}$ representation with the degrees of freedom of 11-dimensional maximal supergravity, only 28 components can be associated to gauge parameters of the theory. The rest correspond to gauge parameters that transform the fields that couple to dual branes. Then, perhaps in this case the closure requires a duality covariant constraint of the form $\omega_{P Q} \xi_{1}^{P} \xi_{2}^{Q}=0$, such that its solutions select a 28-dimensional section of the parameter space, which would cancel the problematic last term in (4.6). Also, it could be possible that even without a constraint of this form, one should supplement the algebra with duality relations between gauge parameters. Analyzing these possibilities lies beyond the scope of this paper, we leave this here as an open problem.

\subsubsection{The $E_{n+1(n+1)}$ case for $n<6$}

We now move to the other exceptional groups. In the case of $E_{n+1(n+1)}$ with $n<6$, the internal $Y$-tensor is symmetric in the pairs of upper and lower indices $Y^{M}{ }_{\left[P^{N}\right.}{ }_{Q]}=0$. Then, in (4.9) the fifth and sixth lines vanish, and the first four determine the gauge transformation of the new components ${ }^{6}$

$$
\begin{array}{r}
Y^{M}{ }_{P}{ }^{N}{ }_{Q} \partial_{N}\left(\hat{\mathcal{L}}_{\hat{\xi}_{1}} \hat{\xi}_{2}\right){ }_{\mu} P Q=Y^{M}{ }_{P}{ }^{N}{ }_{Q} \partial_{N}\left[L_{\hat{\xi}_{1}} \hat{\xi}_{2 \mu}{ }^{P Q}+2 \hat{\xi}_{2}^{\rho} \partial_{[\mu} \hat{\xi}_{1 \rho]}{ }^{P Q}+2 \partial_{\mu} \hat{\xi}_{1}^{Q} \hat{\xi}_{2}^{P}\right. \\
\left.-2 Y^{P}{ }_{R}{ }^{T}{ }_{S} \hat{\xi}_{[2}^{Q} \partial_{T} \hat{\xi}_{1] \mu}{ }^{R S}+\left(\mathcal{L}_{\hat{\xi}_{1}} \hat{\xi}_{2 \mu}{ }^{P Q}-Y^{P}{ }_{R}{ }^{T}{ }_{S} \hat{\xi}_{1}^{Q} \partial_{T} \hat{\xi}_{2 \mu}{ }^{R S}\right)\right]
\end{array}
$$

Notice that this expression is projected by the "intertwining" operator ${ }^{7}$

$$
\frac{1}{2} Y^{M}{ }_{P}^{N}{ }_{Q} \partial_{N}(\ldots)^{P Q}
$$

$$
\begin{aligned}
& { }^{6} \text { We used the identity } \\
& \qquad \begin{aligned}
Y^{M}{ }_{P}{ }^{N} Q_{Q}( & \left.Y^{P}{ }_{R}{ }^{T}{ }_{S} \hat{\xi}_{1}^{Q} \hat{\xi}_{2 \mu}{ }^{R S}-\hat{\xi}_{1}^{T} \hat{\xi}_{2 \mu}{ }^{P Q}\right) \partial_{N} \hat{\varphi}^{\mu} \\
= & Y^{M}{ }_{P}{ }^{N}{ }_{Q} \xi_{1}^{Q}\left(Y^{P}{ }_{R}{ }^{T}{ }_{S} \partial_{T} \hat{\xi}_{2 \mu}{ }^{R S}\right)-\mathcal{L}_{\xi_{1}}\left(Y^{M}{ }_{P}{ }^{N}{ }_{Q} \hat{\xi}_{2 \mu}{ }^{P Q}\right) \\
& -Y^{T}{ }_{R}{ }^{N}{ }_{S} \hat{\xi}_{2 \mu}{ }^{R S} \partial_{T} \hat{\xi}_{1}^{M}+Y^{N}{ }_{P}{ }^{T}{ }_{Q} \partial_{T} \hat{\xi}_{1}^{Q} Y^{M}{ }_{R}{ }_{R}{ }_{S} \hat{\xi}_{2 \mu}{ }^{R S} \\
& +2 \partial_{T} \hat{\xi}_{1}^{Q} \hat{\xi}_{2 \mu}{ }^{R S}\left(Y^{M}{ }_{Q}{ }^{(N \mid}{ }_{P} Y^{P}{ }_{R}{ }^{\mid T)}{ }_{S}-Y^{M}{ }_{R}{ }_{R}\left({ }_{S} \delta_{Q}^{T)}\right)\right. \\
& \left.-4 \partial_{T} \hat{\xi}_{1}^{Q} \hat{\xi}_{2 \mu}{ }^{R S} Y^{M}{ }_{[Q}{ }^{(N \mid}{ }_{P]} Y^{P}{ }_{R}{ }^{\mid T)}{ }_{S}\right] \partial_{N} \hat{\varphi}^{\mu}
\end{aligned}
\end{aligned}
$$

where the last three lines contain section condition-like terms that contribute to (4.10) to form closure constraints that compactify to the quadratic constraints in gauge maximal supergravity, and terms antisymmetric in $Y$ that are irrelevant in this subsection.

${ }^{7}$ We call it intertwining operator since it plays the same projection role as the intertwining tensors (3.32) in the compactified case. 
We can then proceed as in the previous section, and remove the projection up to terms that vanish under it

$$
\begin{aligned}
\left(\hat{\mathcal{L}}_{\hat{\xi}_{1}} \hat{\xi}_{2}\right)_{\mu}<M N>= & L_{\hat{\xi}_{1}} \hat{\xi}_{2 \mu}<M N>+2 \hat{\xi}_{2}^{\rho} \partial_{[\mu} \hat{\xi}_{1 \rho]}<M N>+2 Y^{M}{ }_{P}{ }^{N}{ }_{Q} \partial_{\mu} \hat{\xi}_{1}^{Q} \hat{\xi}_{2}^{P}+\hat{\Gamma}_{\mu}<M N> \\
& -2 Y^{M}{ }_{P}{ }^{N}{ }_{Q} \hat{\xi}_{[2}^{Q} \partial_{T} \hat{\xi}_{1] \mu}<P T> \\
& +\mathcal{L}_{\hat{\xi}_{1}} \hat{\xi}_{2 \mu}<M N>-Y^{M}{ }_{P}{ }^{N}{ }_{Q} \hat{\xi}_{1}^{Q} \partial_{T} \hat{\xi}_{2 \mu}<P T>
\end{aligned}
$$

We are using the notation that

$$
\xi_{\mu}<M N>=Y^{M}{ }_{P}^{N}{ }_{Q} \xi_{\mu}{ }^{P Q}
$$

and have then included $\hat{\Gamma}_{\mu}<M N>$ which satisfies the relations

$$
\partial_{N} \hat{\Gamma}_{\mu}^{<M N>}=0, \quad \hat{\Gamma}_{\mu}^{<M N>} \partial_{N} \hat{\varphi}=0
$$

where $\hat{\varphi}$ represents any field or gauge parameter. As happens in the reduced case, $\hat{\Gamma}_{\mu}<M N>$ must be determined by demanding closure for this new component

$$
\left(\hat{\Delta}_{\hat{\xi}_{1}} \hat{\mathcal{L}}_{\hat{\xi}_{2}} \hat{V}\right)_{\mu}{ }^{<M N>}=0
$$

and this will require the new contributions to include the gauge parameters components of the next step of the hierarchy, and so on.

Notice that the last two terms in (4.26) compactify to the $\left(E_{n+1(n+1)}, n<6\right.$ analog of the) first term in the fifth line of (3.31), which is proportional to the intertwining tensor of the second level. Since the second term in that line is also proportional to this intertwining tensor (contracted with the new 2-form component of the gauge parameters), we can use the last two terms in (4.26) to determine what the next intertwining operator will be. After some algebra one can show that for a symmetric $Y$-tensor, the following identity holds

$$
\begin{aligned}
& Y_{P}^{M}{ }_{P}^{N}\left(Y_{R}^{P}{ }_{R}^{T} \hat{\xi}_{1}^{Q} \partial_{T} \hat{\xi}_{2 \mu}^{R S}-\mathcal{L}_{\hat{\xi}_{1}} \hat{\xi}_{2 \mu}{ }^{P Q}\right) \\
& \quad=\partial_{T}\left(\xi_{1}^{Q} \xi_{2 \mu}{ }^{R S}\right) \tilde{Y}^{M N T}{ }_{Q R S}-3 \xi_{2 \mu}{ }^{R S} \partial_{T} \xi_{1}^{Q} \tilde{Y}^{M N T}{ }_{(Q R S)}
\end{aligned}
$$

where we have defined

$$
\tilde{Y}^{M N T}{ }_{Q R S}=Y^{M}{ }_{P}{ }_{Q} Y^{P}{ }_{R}^{T}{ }_{S}-Y^{M}{ }_{R}{ }^{N}{ }_{S} \delta_{Q}^{T}
$$

The last term in (4.30) vanishes in the cases $E_{n+1(n+1)}$ for $n<5$ [22], but not for $n=5$. Then, at least for $n<5$ and based on (4.7) one can conjecture that the $\hat{\Gamma}_{\mu}\langle M N>$ will contain contributions of the form

$$
\hat{\Gamma}_{\mu}<M N>=\frac{1}{3}\left(\xi_{2}^{\rho} \partial_{T} \xi_{1 \mu \rho}<M N T>+\xi_{2 \mu \rho}<M N T>\partial_{T} \xi_{1}^{\rho}\right)+\ldots
$$

where

$$
\xi_{\mu \nu}<M N T>=\tilde{Y}^{M N T}{ }_{Q R S} \xi_{\mu \nu} Q R S
$$

and that the intertwining operator of the following level will read

$$
\frac{1}{3} \tilde{Y}^{M N T}{ }_{Q R S} \partial_{T}(\ldots)^{Q R S}
$$


Notice that in the $\mathrm{O}(n, n)$ case, the $\tilde{Y}$-tensor vanishes, and this explains why the tensor hierarchy ends at the 1 -form.

Let us note that the $Y$-tensor selects the correct representations for the 1-form vectors. For the different U-duality groups $E_{n+1(n+1)}$ it reads [22]

$$
\begin{array}{ll}
n=2: & Y^{i \alpha}{ }_{l \delta}^{j \beta}{ }_{k \gamma}=4 \delta_{k l}^{i j} \delta_{\gamma \delta}^{\alpha \beta} \\
n=3: & Y^{M{ }_{P}{ }^{N}}{ }_{Q}=\epsilon^{i M N} \epsilon_{i P Q} \\
n=4: & Y^{M}{ }_{P}{ }_{Q}=\frac{1}{2}\left(\gamma^{i}\right)^{M N}\left(\gamma_{i}\right)_{P Q} \\
n=5: & Y^{M}{ }_{P}{ }^{N}{ }_{Q}=10 d^{M N R} \bar{d}_{P Q R}
\end{array}
$$

where for $n=2$, the $\mathrm{SL}(3)$ indices take values $i, j=1,2,3$ and the $\mathrm{SL}(2)$ indices take values $\alpha, \beta=1,2$, for $n=3$ the $\mathrm{SL}(5)$ indices take values $i=1, \ldots, 5$ and $M=[i j]$, for $n=4$ the $\gamma$-matrices correspond to the $16 \times 16 \mathrm{MW}$ representation of $\mathrm{SO}(5,5)$, so $i=1, \ldots, 10$, and for $n=5$ the $d$-tensor is the symmetric invariant of $E_{6(6)}$. Notice that this tensor projects the 1-form components of the gauge parameters to the following representations

$$
Y^{M}{ }_{P}{ }^{N}{ }_{Q} \xi_{\mu}{ }^{P Q}=\left\{\begin{array}{lr}
n=2: 4 \xi_{\mu}{ }^{[i j][\alpha \beta]} & (\mathbf{3}, \mathbf{1}) \\
n=3: \epsilon^{i M N} \xi_{\mu i} & \mathbf{5} \\
n=4: \frac{1}{2}\left(\gamma^{i}\right)^{M N} \xi_{\mu i} & \mathbf{1 0} \\
n=5: 10 d^{M N R} \xi_{\mu R} & \overline{\mathbf{2 7}} \\
n=6:-12\left(t_{\alpha}\right)^{M N} \xi_{\mu}{ }^{\alpha}+\frac{1}{2} \omega^{M N} \xi_{\mu} & \mathbf{1 3 3}+\mathbf{1}
\end{array}\right.
$$

Accordingly, we expect the $\tilde{Y}$-tensor (4.31) to be related to the representations of the 2-form gauge parameters.

In the $E_{6(6)}$ case the obstruction is related to the fact that there is no clear uplift for the $\left(E_{6(6)}\right.$ analog of the) last component of the fifth line in (3.31). This obstruction in the second level of $E_{6(6)}$ has the same origin of the obstruction at the first level of $E_{7(7)}$, discussed around equation (4.22), and the way to circumvent them should proceed in the same way as in the first level of $E_{7(7)}$. Notice however that since the first level of the algebra closes in this case, one can write an action that includes the 2 -form curvature of the gauge fields, as in $[50,51]$.

Let us now introduce the field degrees of freedom and compute the fluxes to see what the implications of this will be in the construction of EFTs. Consider a generalized fielddependent frame, in the spirit of (3.34)

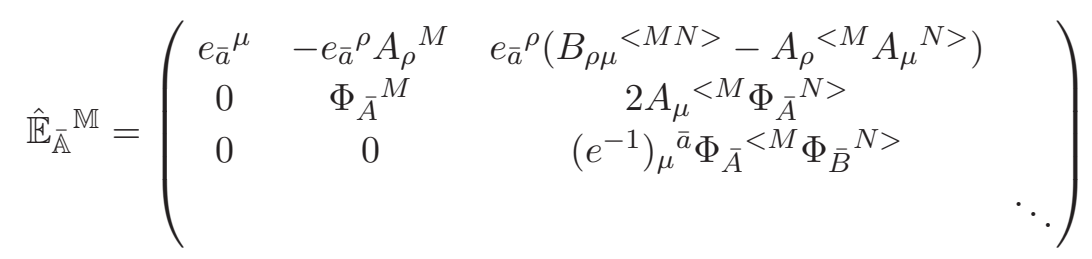


and define the generalized fluxes as

$$
\hat{\mathbb{F}}_{\overline{\mathbb{A}} \overline{\mathbb{B}}} \overline{\mathbb{C}}=\left(\hat{\mathcal{L}}_{\hat{\mathbb{E}}_{\overline{\mathbb{A}}}} \hat{\mathbb{E}}_{\overline{\mathbb{B}}}\right)^{\mathbb{M}}\left(\hat{\mathbb{E}}^{-1}\right)_{\mathbb{M}} \overline{\mathbb{C}}
$$

Just to put an example, they contain the 2-form curvature for the gauge fields

$$
\hat{\mathbb{F}}_{\bar{a} \bar{b}}^{\bar{C}}=-e_{\bar{a}}^{\mu} e_{\bar{b}}^{\nu}\left(\Phi^{-1}\right)_{M}{ }^{\bar{C}} \mathcal{F}_{\mu \nu}{ }^{M}
$$

with

$$
\mathcal{F}_{\mu \nu}{ }^{M}=2 \partial_{[\mu} A_{\nu]}{ }^{M}-\left[\left[A_{\mu}, A_{\nu}\right]\right]^{M}-\frac{1}{2} Y^{M}{ }_{P}{ }^{N}{ }_{Q} \partial_{N} B_{\mu \nu}{ }^{P Q}
$$

where we have defined the internal exceptional C-bracket

$$
\left[\left[A_{\mu}, A_{\nu}\right]\right]=\frac{1}{2}\left(\mathcal{L}_{A_{\mu}} A_{\nu}-\mathcal{L}_{A_{\nu}} A_{\mu}\right)
$$

and assumed that the $Y$-tensor is symmetric. Since, at this level the algebra closes for $E_{n+1(n+1)}, n<6$, it is possible to define a theory in terms of this curvature, as achieved in $[50,51]$ for $E_{6(6)}$.

The generalized frame above was written in a gauged fixed $H=\mathrm{SO}(1, d-1) \times H_{i}$ triangular form $\left(H_{i}\right.$ is the local compact maximal subgroup of the U-duality group). Then, in order to analyze the gauge transformations of its components, we must make sure that the generalized diffeomorphic transformations preserve this gauge choice, by performing a compensating $H$-transformation. ${ }^{8}$ The combined transformations that preserve the gaugedfixed form of the generalized frame read ${ }^{9}$

$$
\delta^{\prime} \hat{\mathbb{E}}_{\overline{\mathbb{A}}}^{\mathbb{M}}=\hat{\mathcal{L}}_{\hat{\xi}} \hat{\mathbb{E}}_{\overline{\mathbb{A}}} \mathbb{M}^{\mathbb{M}}-\Lambda_{\overline{\mathbb{A}}} \overline{\mathbb{B}}_{\mathbb{E}_{\bar{B}}} \mathbb{M}
$$

We will focus for simplicity in the transformation of the vielbein $e_{\bar{a}}{ }^{\mu}$, gauge fields $A_{\mu}{ }^{M}$ and scalars $\Phi_{\bar{A}}{ }^{M}$, but this analysis can be extended to the other components. In particular, demanding that the ()$_{21}$ component remains zero under a gauge transformation selects

$$
\Lambda_{\bar{A}}^{\bar{b}}=-\left(e^{-1}\right)_{\mu}{ }^{\bar{b}} \Phi_{\bar{A}}{ }^{P} \partial_{P} \hat{\xi}^{\mu}, \quad \Lambda_{\bar{a}}^{\bar{B}}=\eta_{\bar{a} \bar{b}} \delta^{\bar{A} \bar{B}}\left(e^{-1}\right)_{\mu}{ }^{\bar{b}} \Phi_{\bar{A}}{ }^{P} \partial_{P} \hat{\xi}^{\mu}
$$

Notice that this compactifies to zero, which is the reason why no compensation of this form was required in the previous section. Here we introduced the components of the invariant $\mathrm{SO}(1, d-1) \times H_{i}$ metric, namely the flat Minkowski and Euclidean metrics $\eta_{\bar{a} \bar{b}}$ and $\delta^{\bar{A}} \bar{B}$. Then, we can readily find the gauge transformations of the components

$$
\begin{aligned}
& \delta^{\prime} e_{\bar{a}}^{\mu}=L_{\hat{\xi}} e_{\bar{a}}^{\mu}+\hat{\xi}^{P} \partial_{P} e_{\bar{a}}^{\mu}+e_{\bar{a}}^{\rho} A_{\rho}^{P} \partial_{P} \hat{\xi}^{\mu} \\
& \delta^{\prime} A_{\mu}{ }^{M}=L_{\hat{\xi}} A_{\mu}{ }^{M}+\partial_{\mu} \hat{\xi}^{M}+\mathcal{L}_{\hat{\xi}} A_{\mu}^{M}-\frac{1}{2} \partial_{N} \hat{\xi}_{\mu}<M N> \\
& -\frac{1}{2}\left(B_{\mu \rho}<M N>-A_{\mu}{ }^{<M} A_{\rho}{ }^{N>}\right) \partial_{N} \hat{\xi}^{\rho}-A_{\mu}{ }^{P} \partial_{P} \hat{\xi}^{\rho} A_{\rho}{ }^{M}+g_{\mu \nu} \mathcal{M}^{M P} \partial_{P} \hat{\xi}^{\nu} \\
& \delta^{\prime} \Phi_{\bar{A}}{ }^{M}=L_{\hat{\xi}} \Phi_{\bar{A}}{ }^{M}+\mathcal{L}_{\hat{\xi}} \Phi_{\bar{A}}{ }^{M}+A_{\rho}{ }^{<M} \Phi_{\bar{A}}{ }^{N>} \partial_{N} \hat{\xi}^{\rho}-A_{\mu}{ }^{M} \Phi_{\bar{A}}{ }^{P} \partial_{P} \hat{\xi}^{\mu}
\end{aligned}
$$

\footnotetext{
${ }^{8}$ Let us emphasize that actually this is not needed in our approach since by construction the frame is indeed a collection of vectors, and therefore its transformation is well defined and independent of the choice of frame.

${ }^{9}$ We note these transformation with $\delta^{\prime}$, to distinguish them from the transformations $\delta$ considered before.
} 
where we have defined the internal "scalar" matrix $\mathcal{M}^{M N}=\Phi_{\bar{A}}{ }^{M} \delta^{\bar{A}} \bar{B} \Phi_{\bar{B}}{ }^{N}$ and the "external" metric $g_{\mu \nu}=\left(e^{-1}\right)_{\mu}{ }^{\bar{a}} \eta_{\bar{a} \bar{b}}\left(e^{-1}\right)_{\nu} \bar{b}$.

For the sake of completeness, we now establish the redefinitions required to make contact with the results in $[50,51]$. Regarding the fluxes, one can already see that the gauge curvature (4.40) coincides. Focussing on the internal transformation of the gauge field $A_{\mu}{ }^{M}$, we find that redefining the 1-form gauge parameter as

$$
\frac{1}{2} \hat{\xi}_{\mu}^{<M N>}=\hat{\Xi}_{\mu}<M N>+\hat{\xi}^{<M} A_{\mu}^{N>}
$$

one obtains

$$
\delta^{\prime} A_{\mu}{ }^{M}=\mathcal{D}_{\mu} \hat{\xi}^{M}-\partial_{N} \hat{\Xi}_{\mu}<M N>, \quad \mathcal{D}_{\mu}=\partial_{\mu}-\mathcal{L}_{A_{\mu}}
$$

which is the internal gauge transformation of the gauge fields as exposed in $[50,51]$. There, instead of considering purely external diffeomorphisms, the authors combined them with a subsector of internal diffeos where the internal gauge parameters are restricted to the form $\hat{\xi}^{M}=-\hat{\xi}^{\nu} A_{\nu}{ }^{M}$, such that this particular combination results in gauge transformations that look like external diffeomorphisms, but are manifestly covariantized with respect to the gauge fields. To make contact with those transformations, one also has to make the following redefinition

$$
\hat{\Xi}_{\mu}<M N>\rightarrow \hat{\Xi}_{\mu}<M N>-\frac{1}{2} \hat{\xi}^{\rho}\left(B_{\mu \rho}<M N>-A_{\mu}<M A_{\rho}^{N>}\right)
$$

and then the gauge transformations (4.44) reduce to

$$
\begin{aligned}
\delta^{\prime} e_{\bar{a}}{ }^{\mu} & =\hat{\xi}^{\rho} \mathcal{D}_{\rho} e_{\bar{a}}{ }^{\mu}-e_{\bar{a}}{ }^{\rho} \mathcal{D}_{\rho} \hat{\xi}^{\mu} \\
\delta^{\prime} A_{\mu}{ }^{M} & =\hat{\xi}^{\rho} \mathcal{H}_{\rho \mu}+g_{\mu \nu} \mathcal{M}^{M P} \partial_{P} \hat{\xi}^{\mu} \\
\delta^{\prime} \Phi_{\bar{A}}{ }^{M} & =\hat{\xi}^{\rho} \mathcal{D}_{\rho} \Phi_{\bar{A}}{ }^{M}
\end{aligned}
$$

One could also extended this analysis for the 2-form field, were we expect the transformation to depend on its curvature, and then it seems that duality relations would be required to make contact with $[50,51]$. It would be interesting to explore the role of dualities of that type at the level of the algebra of generalized diffeomorphisms discussed here.

\subsection{The gauge structure of the full generalized Lie derivative}

Collecting the information from the previous subsections, we can now try to condense the notation. As in the compactified case, the gauge parameters can be thought of as components of a generalized gauge parameter in an extended mega-space

$$
\hat{\xi}^{\mathbb{M}}=\left(\hat{\xi}^{\mu}, \hat{\xi}^{M}, \hat{\xi}_{\mu}^{<M N>}, \hat{\xi}_{\mu \nu}^{<M N P>}, \ldots\right)
$$

One can then define a generalized Lie derivative that takes the form

$$
\left(\hat{\mathcal{L}}_{\hat{\xi}_{1}} \hat{\xi}_{2}\right) \hat{\mathbb{M}}^{\mathbb{M}}=\hat{\xi}_{1}^{\mathbb{P}} \partial_{\mathbb{P}} \hat{\xi}_{2}^{\mathbb{M}}-\hat{\xi}_{2}^{\mathbb{P}} \partial_{\mathbb{P}} \hat{\xi}_{1}^{\mathbb{M}}+Y^{\mathbb{M}} \mathbb{P}_{\mathbb{Q}}^{\mathbb{N}} \partial_{\mathbb{N}} \hat{\xi}_{1}^{\hat{Q}} \hat{\xi}_{2}^{\mathbb{P}}
$$

The derivatives are restricted to only have components $\partial_{\mathbb{M}}=\left(\partial_{\mu}, \partial_{M}, 0, \ldots\right)$. One could include derivatives with respect to all the coordinates, and impose a generalized section 
condition (this is what happens in the DFT T-duality case, where the derivatives in the directions of the 1-form gauge parameters $\partial^{\mu}$ are included for full covariance of the theory, and then removed through a section condition). The $Y$-tensor contains GL( $d)$ and $E_{n+1(n+1)}$ invariants, and when it is restricted to the internal sector it coincides with the Y-tensors defined in (2.7) and (4.35). The purely external components of it vanish, and then one recovers standard Riemannian geometry in the purely external sector.

In components, this generalized Lie derivative takes the form

$$
\begin{aligned}
& \left(\hat{\mathcal{L}}_{\hat{\xi}_{1}} \hat{\xi}_{2}\right)^{\mu}=L_{\hat{\xi}_{1}} \hat{\xi}_{2}^{\mu}+\hat{\xi}_{1}^{P} \partial_{P} \hat{\xi}_{2}^{\mu}-\hat{\xi}_{2}^{P} \partial_{P} \hat{\xi}_{1}^{\mu} \\
& \left(\hat{\mathcal{L}}_{\hat{\xi}_{1}} \hat{\xi}_{2}\right)^{M}=L_{\hat{\xi}_{1}} \hat{\xi}_{2}^{M}-\hat{\xi}_{2}^{\rho} \partial_{\rho} \hat{\xi}_{1}^{M}+\mathcal{L}_{\hat{\xi}_{1}} \hat{\xi}_{2}^{M}+\frac{1}{2}\left(\partial_{N} \hat{\xi}_{1 \rho}<M N>\hat{\xi}_{2}^{\rho}+\hat{\xi}_{2 \rho}<M N>\partial_{N} \hat{\xi}_{1}^{\rho}\right) \\
& \left(\hat{\mathcal{L}}_{\hat{\xi}_{1}} \hat{\xi}_{2}\right)_{\mu}<M N>=L_{\hat{\xi}_{1}} \hat{\xi}_{2 \mu}<M N>-2 \hat{\xi}_{2}^{\rho} \partial_{[\rho} \hat{\xi}_{1 \mu]}<M N>+Y^{M}{ }_{P}{ }^{N}{ }_{Q}\left(2 \partial_{\mu} \hat{\xi}_{1}^{Q} \hat{\xi}_{2}^{P}-\hat{\xi}_{2}^{Q} \partial_{T} \hat{\xi}_{1 \mu}<P T>\right) \\
& +\mathcal{L}_{\hat{\xi}_{1}} \hat{\xi}_{2 \mu}<M N>+\frac{1}{3}\left(\xi_{2}^{\rho} \partial_{T} \xi_{1 \mu \rho}<M N T>+\xi_{2 \mu \rho}<M N T>\partial_{T} \xi_{1}^{\rho}\right)
\end{aligned}
$$

We have only worked the hierarchy up to this level, but the computations can be pushed forward with more effort. The closure of the external component is achieved up to terms that would vanish under imposing the section condition (and compactify to zero). The first level internal component includes terms that are projected by the first intertwining operator

$$
Y^{M}{ }_{P}^{N}{ }_{Q} \partial_{N}(\ldots)^{P Q}=\partial_{N}(\ldots)^{<M N>}
$$

and closes up to section-condition-like terms (that compactify to quadratic constraints) and terms proportional to $Y^{M}{ }_{\left[P^{N}\right.}{ }_{Q]}$. While this vanishes when the U-duality group is $E_{n+1(n+1)}$ with $n<6$, we find a closure obstruction for $E_{7(7)}$, on which we commented in subsection 4.2.2. Regarding the second level component $\hat{\xi}_{\mu}\langle M N\rangle$, it includes in its transformation terms that are projected by the second intertwining operator

$$
\tilde{Y}^{M N T}{ }_{Q R S} \partial_{T}(\ldots)^{Q R T}=\partial_{T}(\ldots)^{<M N T>}
$$

The closure obstruction in this case is proportional to $\tilde{Y}^{M N T}{ }_{(Q R S)}$. Now this vanishes for $n<5$, but not for $E_{6(6)}$, and this obstruction was commented in subsection 4.2.3. We expect issues like this will appear at level $7-n$ for $E_{n+1(n+1)}$, for the adjoint representations, but this has to be explored further.

We have also shown how to introduce a field-dependent generalized frame and how to extract the gauge transformations on its components, and how to build the covariant quantities. These results will be useful when constructing full duality invariant Exceptional Field Theories.

\section{Contact with 11-dimensional supergravity (and beyond)}

In this section we concentrate on $d=4$ and U-duality group $E_{7(7)}$. Using a GL(7) $\subset$ $\mathrm{SL}(8) \subset E_{7}$ decomposition, were GL(7) acts on the "internal" tangent space in the supergravity limit, i.e. on the coordinates dual to momentum, which can be either defined by 
hand, or by a section condition. By this, we give the 11-dimensional origin of the gauge parameters, external 1-forms and some of the 2 -forms, etc. that we have seen. The same can be done for type II theories, but we will skip it (all the information about the purely internal objects can be found for example in [19]).

The exceptional generalized tangent space encodes all the gauge transformations of 11-dimensional supergravity, namely diffeomorphisms and gauge transformations of the 3 -form field. In order to recover an electro-magnetic covariant formulation, we need to include their duals, namely a 5 -form corresponding to gauge transformations of the 6 -form potential, and a gauge transformation for a "dual graviton", transforming as a 7 -form times a 1-form [16]. The generalized tangent space can equivalently be constructed by counting the charges of the theory, namely momentum, M2 and M5 brane charge, and KK monopole charge (being the electric charge for the dual graviton). Furthermore, to build the tensor hierarchy, or equivalently to go higher levels, more brane charges are needed, corresponding to the so-called "U-branes" or "exotic branes" [59]-[61] (i.e. configurations with non-trivial U-duality monodromies). For example, to reconstruct the 133 space-time 1 -forms, we need to add the exotic branes termed $5^{3}$ and $2^{6}$ in [61] (see [59] for an earlier discussion). These have no direct 11-dimensional interpretation, as they exist only in spaces with 3 and 6 isometries respectively, and are obtained from the type IIA NS5 brane by a chain of dualities and uplift. They transform as an 8-form tensor, a 3-form and 6-form respectively, and appear already when counting scalar degrees of freedom in $E_{8(8)}$ [29]. The generalized tangent space is therefore locally

$$
\begin{array}{ccccccc}
E= & \oplus \Lambda^{2} T^{*} & \oplus \Lambda^{5} T^{*} & \oplus \Lambda^{7} T^{*} \otimes T^{*} & \oplus \Lambda^{8} T^{*} \otimes \Lambda^{3} T^{*} & \oplus \Lambda^{8} T^{*} \otimes \Lambda^{6} T^{*} & \oplus \cdots \\
p & M 2 & M 5 & K K & 5^{3} & 2^{6} & \cdots
\end{array}
$$

where $T$ is 11-dimensional. The dots account for higher p-form representations. For example, to obtain the 912 degrees of freedom that appear in space-time 2-forms, we need extra forms transforming as 9 -forms times some lower form. These show up in the $l_{1}$ representation of $E_{11}$ at fourth order [31-36].

Splitting $T=T_{4}+T_{7}$ we get the GL(4) and GL(7) representations indicated in table $1 .{ }^{10}$

The bosonic fields of 11-dimensional supergravity are the metric and the 3-form gauge field. Again, in order to achieve 4-dimensional electro-magnetic invariance, we need to include their duals, a 6 -form field $A_{6}$ and the dual graviton $B$, transforming as $\Lambda^{8} T^{*} \otimes$ $T^{*}$ [29]. The "gauge field" whose electric charge are the exotic branes included so far, termed $A_{9^{3}}$ and $A_{9^{6}}$, should transform respectively as $\Lambda^{9} T^{*} \otimes \Lambda^{3} T^{*}$ and $\Lambda^{9} T^{*} \otimes \Lambda^{6} T^{*}$. Again, to fill out the corresponding $E_{7(7)}$ representations at the level of space-time 3 -forms

\footnotetext{
${ }^{10}$ There is a subtlety in counting degrees of freedom: the "dual branes" (KK, $5^{3}, 2^{6}$, etc) exist only in spaces with $\mathrm{U}(1)$ isometries. Their respective $T^{*}, \Lambda^{3} T^{*}$ and $\Lambda^{6} T^{*}$ lie along these compact directions. We have therefore excluded the possibility of placing 4-dimensional space-time indices along them. If on the contrary we allow this possibility, the KK monopole would count as 50 1-form degrees of freedom, and the total number of 1 -forms would be $133+1$, in accordance with the $E_{11}$ results [31-36], while the total number of 2 -forms would be $912+56$.
} 


\begin{tabular}{|c|ccccccc|c|c|}
\hline & $p$ & $M 2$ & $M 5$ & $\begin{array}{l}K K \\
\mathrm{GL}(4)\end{array}$ & $5^{3}$ & $2^{6}$ & $\cdots$ & total & $E_{7(7)}$ repr \\
\hline scalar & 7 & 21 & 21 & 7 & 0 & 0 & 0 & 56 & $\xi^{M} \in \mathbf{5 6}$ \\
1-form & 0 & 7 & 35 & 49 & 35 & 7 & 0 & 133 & $\xi_{\mu}^{\alpha} \in \mathbf{1 3 3}$ \\
2-form & 0 & 1 & 35 & 147 & 245 & 49 & $\cdots$ & $477+\cdots$ & $\xi_{\mu \nu}^{\mathcal{A}} \in \mathbf{9 1 2}$ \\
3-form & 0 & 0 & 21 & 245 & 735 & 147 & $\cdots$ & $1148+\cdots$ & $\xi_{\mu \nu \rho}{ }^{\mathbf{A}} \in \mathbf{1 3 3}+\mathbf{8 6 4 5}$ \\
\hline
\end{tabular}

Table 1. GL(4) and GL(7) decomposition of the generalized tangent space. We give the number of internal components.

\begin{tabular}{|c|ccccccc|c|c|}
\hline GL(4) & $g$ & $A_{3}$ & $A_{6}$ & $\begin{array}{c}B \\
\mathrm{GL}(7)\end{array}$ & $A_{9^{3}}$ & $A_{9^{6}}$ & $\cdots$ & total & $E_{7(7)}$ repr \\
\hline scalar & 28 & 35 & 7 & 0 & 0 & 0 & 0 & 70 & $\Phi_{\bar{A}}{ }^{M} \in \mathbf{1 3 3}$ \\
1-form & 7 & 21 & 21 & 7 & 0 & 0 & 0 & 56 & $A_{\mu}{ }^{M} \in \mathbf{5 6}$ \\
2-form & 0 & 7 & 35 & 49 & 35 & 7 & 0 & 133 & $B_{\mu \nu}{ }^{\alpha} \in \mathbf{1 3 3}$ \\
3-form & 0 & 1 & 35 & 147 & 245 & 49 & $\cdots$ & $477+\cdots$ & $C_{\mu \nu \rho}{ }^{\mathcal{A}} \in \mathbf{9 1 2}$ \\
4-form & 0 & 0 & 21 & 245 & 735 & 147 & $\cdots$ & $1148+\cdots$ & $D_{\mu \nu \lambda \rho}{ }^{\mathbf{A}} \in \mathbf{1 3 3}+\mathbf{8 6 4 5}$ \\
\hline
\end{tabular}

Table 2. GL(4) and GL(7) decomposition of the bosonic fields. $B$ represents the dual graviton, and $A_{9^{3}}, A_{9^{6}}$ the gauge fields that couple electrically to the $5^{3}$ and $2^{6}$ branes.

and on, we need to include higher p-forms. The GL(4) and GL(7) representations we obtain for the bosonic fields are shown in table 2 .

It will be useful to find the embedding of the metric and 3-form field, as well as their gauge transformations (diffeomorphisms and 2-forms) in terms of $\mathrm{SL}(8)$ representations. For that, we use the following notation: $a, b, \ldots=1, \ldots, 8$ are indices in the fundamental of SL(8), while $m, n=1, \ldots, 7$ are in the fundamental of GL(7). Later on we will use $\tilde{M}=1, \ldots, 11$ to denote indices along eleven-dimensional space-time (i.e. $\tilde{M}=(\mu, m)$ ).

The fundamental representation of $E_{7(7)}$ decomposes with respect to SL(8) into

$$
\begin{aligned}
\mathbf{5 6} & =\mathbf{2 8}+\mathbf{2 8}^{\prime} \\
\xi & =\left(\xi^{a b}, \xi_{a b}\right)
\end{aligned}
$$

and the adjoint

$$
\begin{aligned}
133 & =63+70 \\
\Phi & =\left(\Phi_{b}^{a}, \Phi_{a b c d}\right)
\end{aligned}
$$

where $\Phi_{a}^{a}=0$ and $\Phi_{a b c d}$ is fully antisymmetric. Finally, the 912 decomposes into

$$
\begin{aligned}
912 & =36+420+36^{\prime}+420^{\prime} \\
F & =\left(F^{a b}, F_{d}^{a b c}, F_{a b}, F_{a b c}{ }^{d}\right)
\end{aligned}
$$

where $F^{b a}=F^{a b}$ and $F_{c}^{a b c}=0$ and similarly for the objects with the indices down. 
We can then embed the generators of diffeomorphisms $v$ and two-form gauge transformations $\lambda_{2}$ of conventional 11-dimensional supergravity, whose different external components are given in the first two columns in table 1 , in the following way

$$
\begin{array}{rlrlrl}
\xi^{m 8}=v^{m}, & \xi_{m n} & =\lambda_{m n} & & \in \mathbf{2 8}+\mathbf{2 8} 8^{\prime} & \in \mathbf{5 6} \\
\xi^{\mu}=v^{\mu}, & \xi_{\mu}{ }^{8} m & =\lambda_{\mu m} & \in \mathbf{1}+\mathbf{6 3} & & \in \mathbf{1}+\mathbf{1 3 3} \\
\xi_{\mu \nu}^{88} & =\lambda_{\mu \nu} & \in \mathbf{3 6} & & \in \mathbf{9 1 2}
\end{array}
$$

The scalar, vector, 2-form and 3-form fields coming from the metric and $A_{3}$ are embedded as

$$
\begin{array}{rlrlrl}
\Phi_{m}{ }^{n}=a_{m}{ }^{n}, & \Phi_{m n p 8} & =A_{m n p} & \in \mathbf{7 0} & \in \mathbf{1 3 3} \\
A_{\mu}{ }^{m 8}=a_{\mu}{ }^{m}, & A_{\mu m n} & =A_{\mu m n} & \in \mathbf{2 8}+\mathbf{2 8}^{\prime} & & \in \mathbf{5 6} \\
B_{\mu \nu}{ }^{8} m & =A_{\mu \nu m} & \in \mathbf{6 3} & & \in \mathbf{1 3 3} \\
C_{\mu \nu \rho}{ }^{88} & =A_{\mu \nu \rho} & \in \mathbf{3 6} & & \in \mathbf{9 1 2}
\end{array}
$$

Given this, the first row of the generalized vielbein (3.34) for 11-dimensional supergravity reads therefore

$$
\begin{aligned}
& \mathbb{E}_{\bar{a}}{ }^{\mathbb{M}}=-e_{\bar{a}}{ }^{\rho}\left(-\delta_{\rho}^{\mu},\left[a_{\rho}{ }^{m}\right]^{8}+A_{\rho m n},\left[A_{\rho \mu m}+a_{\rho}{ }^{p} A_{p \mu m}\right]^{8},\right. \\
& {\left[A_{\mu \nu \rho}+a_{\rho}^{p} A_{\mu \nu p}+\frac{1}{3} a_{\rho}^{p} a_{\mu}^{q} A_{\nu p q}\right]^{88}, } \\
& {\left.\left[A_{\rho[m n} A_{p] \mu \nu}+\frac{1}{3} a_{\rho}^{q} A_{q \mu[m} A_{n p] \nu}+\frac{1}{3} A_{\rho m n} a_{[\mu}{ }^{q} A_{\nu] p q}\right]^{8}\right), }
\end{aligned}
$$

where the superindex 8 completes the SL(8) representation (for example the second term corresponds to $\mathbb{M}={ }^{m 8}$ ).

One can now use the generalized Lie derivative (4.7) with parameters (5.5), applied to this generalized vielbein and obtain the gauge transformations of the fields in 11-dimensional supergravity. After some algebra, one can show that from $\left(\hat{\mathcal{L}}_{\hat{\xi}} \hat{\mathbb{E}}_{\bar{a}}\right)^{\mu}$ and $\left(\hat{\mathcal{L}}_{\hat{\xi}} \hat{\mathbb{E}}_{\bar{a}}\right)^{M}$ one recovers precisely the gauge transformations of 11-dimensional supergravity, namely

$$
\begin{aligned}
\delta e_{\bar{a}}{ }^{\tilde{M}} & =\tilde{L}_{v} e_{\bar{a}}^{\tilde{M}} \\
\delta A_{\mu m n} & =\tilde{L}_{v} A_{\mu m n}+(\mathrm{d} \lambda)_{\mu m n}
\end{aligned}
$$

where $\tilde{L}_{v}$ means the ordinary Lie derivative with parameter $v^{\tilde{M}}=\left(v^{\mu}, v^{m}\right)$, and we have used $e_{\bar{a}}^{m}=e_{\bar{a}}^{\rho} a_{\rho}^{m}$.

Note that we have correctly reproduced the gauge transformations of 11-dimensional supergravity using the generalized Lie derivative (4.51) which, as we have shown, does not close at the first level for $E_{7(7)}$. This is so because the obstruction for closure goes away when the gauge parameters and the fields are those of 11-dimensional supergravity. Indeed, on the one hand they obviously satisfy the section condition. On the other, terms involving $Y^{M}{ }_{\left[P^{N}\right.}{ }_{Q]}=\frac{1}{2} \omega^{M N} \omega_{P Q}$ go away when contracted with gauge fields or gauge parameters, 
as the only non-zero components of the latter are those with fundamental indices $P$ or $Q={ }^{m 8}$ or $P={ }_{m n}$, and therefore vanish in the symplectic products.

Furthermore, it is not hard to show that restricting the generalized tangent space to vectors and two-forms, i.e. for $U=(u, \lambda), V=(v, \omega), u, v \in T, \lambda, \omega \in \Lambda^{2} T^{*}$, the generalized Lie derivative

$$
\mathcal{L}_{U} V=\left(L_{u} v, L_{u} \omega-\iota_{v} \mathrm{~d} \lambda\right)
$$

closes without any need to introduce other forms.

\section{Conclusions}

We have addressed the construction of U-duality invariant generalized diffeomorphisms for M-theory. Taking as a starting point the generalized Lie derivatives for the different Uduality groups introduced in [20-22], we explored the completion to the full external plus internal space-time.

We began with the Scherk-Schwarz-type compactification ansatz leading to gauged maximal supergravity, and specialized the analysis to the $E_{7(7)}$ case in 4-dimensions in section 3. Closure of the algebra is highly non-trivial and requires an extension of the tangent space to include the so-called tensor hierarchy. The closure at each level of the hierarchy dictates the transformation rules of the following level. There is also a hierarchy of intertwining tensors, such that when a given level of the hierarchy is projected by the corresponding intertwiner, the (projected) sub-algebra formed by it and the previous levels closes. Interestingly, this procedure allowed us to build a full generalized Lie derivative in the reduced case, that encodes all the gauge transformations of maximal supergravity and the tensor hierarchy (3.30)-(3.31).

The field content is introduced through a generalized frame (3.34), in such a way that the different components transform appropriately (3.35). This is a novel construction, in which the different levels of the tensor hierarchy are embedded into different components of a generalized frame, in analogy to what happens with the 2-form in DFT. We then define generalized fluxes in terms of the generalized frame and Lie derivative (3.36), and show that the different components correspond to covariant quantities in the theory (3.37): curvatures, covariant derivatives, etc. Since the generalized Lie derivative closes, the closure of the generalized fluxes define the BIs of the theory (3.39).

Encouraged by this construction, we then moved towards a full generalized Lie derivative on the extended space (4.50). Again, closure requires an extended tangent space to accommodate the tensor hierarchy, and level by level the closure conditions dictate the transformation rules of the next level. We identified the "intertwining operators" of the first levels, which play a role analog to (and compactify to) the intertwining embedding tensor in the reduced theory.

Intriguingly, we found closure obstructions at different levels of the hierarchy for different U-duality groups. Although we have commented on how they can be circumvented, this issue deserves further study.

Let us finally comment on future lines of investigation. Regarding the reduced case of section 3, it would be interesting to construct a generalized geometry through covariant 
derivatives, connections, torsion and curvatures. This is expected to give rise to an action and equations of motion of a democratic formulation of maximal gauged supergravity. This framework can also be used to explore non-geometry, and possible uplifts for the new maximal gauged supergravities in [68]. Regarding the extended construction of section 4, it would be interesting to further explore mechanisms to achieve full closure, and apply the generalized frame and flux techniques of section 3 to build covariant quantities and actions of Exceptional Field Theories. A construction of an underlying generalized geometry with generalized connections and curvatures would allow to systematically obtain the actions and equations of motion.

\section{Acknowledgments}

We thank B. de Witt, J. Palmkvist, M. Shigemori, D. Waldram and specially A. Coimbra for useful discussions and comments. G. A. thanks the ICTP and CERN for hospitality during the completion of this work. D. M. and J. A. R. thank Institut de Physique Théorique, CEA/Saclay, for hospitality. This work was partially supported by EPLANET, CONICET, PICT-2012-513 and the ERC Starting Independent Researcher Grant 259133ObservableString.

Open Access. This article is distributed under the terms of the Creative Commons Attribution License (CC-BY 4.0), which permits any use, distribution and reproduction in any medium, provided the original author(s) and source are credited.

\section{References}

[1] W. Siegel, Superspace duality in low-energy superstrings, Phys. Rev. D 48 (1993) 2826 [hep-th/9305073] [INSPIRE].

[2] C. Hull and B. Zwiebach, Double field theory, JHEP 09 (2009) 099 [arXiv:0904.4664] [INSPIRE].

[3] C. Hull and B. Zwiebach, The gauge algebra of double field theory and Courant brackets, JHEP 09 (2009) 090 [arXiv:0908.1792] [INSPIRE].

[4] O. Hohm, C. Hull and B. Zwiebach, Background independent action for double field theory, JHEP 07 (2010) 016 [arXiv: 1003.5027] [INSPIRE].

[5] O. Hohm, C. Hull and B. Zwiebach, Generalized metric formulation of double field theory, JHEP 08 (2010) 008 [arXiv: 1006.4823] [INSPIRE].

[6] O. Hohm and S.K. Kwak, Frame-like geometry of double field theory, J. Phys. A 44 (2011) 085404 [arXiv: 1011.4101] [inSPIRE].

[7] G. Aldazabal, D. Marques and C. Núñez, Double field theory: a pedagogical review, Class. Quant. Grav. 30 (2013) 163001 [arXiv:1305.1907] [INSPIRE].

[8] D.S. Berman and D.C. Thompson, Duality symmetric string and M-theory, arXiv:1306.2643 [INSPIRE].

[9] O. Hohm, D. Lüst and B. Zwiebach, The spacetime of double field theory: review, remarks and outlook, Fortsch. Phys. 61 (2013) 926 [arXiv:1309.2977] [INSPIRE]. 
[10] N. Hitchin, Generalized Calabi-Yau manifolds, Quart. J. Math. Oxford Ser. 54 (2003) 281 [math/0209099] [INSPIRE].

[11] M. Gualtieri, Generalized complex geometry, math/0401221 [INSPIRE].

[12] A. Coimbra, C. Strickland-Constable and D. Waldram, Supergravity as generalised geometry I: type II theories, JHEP 11 (2011) 091 [arXiv:1107.1733] [INSPIRE].

[13] P. Koerber, Lectures on generalized complex geometry for physicists, Fortsch. Phys. 59 (2011) 169 [arXiv:1006.1536] [InSPIRE].

[14] M. Graña, R. Minasian, M. Petrini and D. Waldram, T-duality, generalized geometry and non-geometric backgrounds, JHEP 04 (2009) 075 [arXiv:0807.4527] [INSPIRE].

[15] C.M. Hull, Generalised geometry for M-theory, JHEP 07 (2007) 079 [hep-th/0701203] [INSPIRE].

[16] P.P. Pacheco and D. Waldram, M-theory, exceptional generalised geometry and superpotentials, JHEP 09 (2008) 123 [arXiv:0804.1362] [INSPIRE].

[17] G. Dall'Agata, N. Prezas, H. Samtleben and M. Trigiante, Gauged supergravities from twisted doubled tori and non-geometric string backgrounds, Nucl. Phys. B 799 (2008) 80 [arXiv: 0712.1026] [INSPIRE].

[18] C. Hillmann, Generalized $E_{7(7)}$ coset dynamics and $D=11$ supergravity, JHEP 03 (2009) 135 [arXiv:0901.1581] [INSPIRE].

[19] G. Aldazabal, E. Andres, P.G. Camara and M. Graña, U-dual fluxes and generalized geometry, JHEP 11 (2010) 083 [arXiv:1007.5509] [INSPIRE].

[20] A. Coimbra, C. Strickland-Constable and D. Waldram, $E_{d(d)} \times \mathbb{R}^{+}$generalised geometry, connections and M-theory, JHEP 02 (2014) 054 [arXiv: 1112.3989] [INSPIRE].

[21] A. Coimbra, C. Strickland-Constable and D. Waldram, Supergravity as generalised geometry II: $E_{d(d)} \times \mathbb{R}^{+}$and M-theory, JHEP 03 (2014) 019 [arXiv: 1212.1586] [INSPIRE].

[22] D.S. Berman, M. Cederwall, A. Kleinschmidt and D.C. Thompson, The gauge structure of generalised diffeomorphisms, JHEP 01 (2013) 064 [arXiv:1208.5884] [INSPIRE].

[23] G. Aldazabal, W. Baron, D. Marques and C. Núñez, The effective action of double field theory, JHEP 11 (2011) 052 [Erratum ibid. 1111 (2011) 109] [arXiv:1109. 0290] [INSPIRE].

[24] D. Geissbuhler, Double field theory and $N=4$ gauged supergravity, JHEP 11 (2011) 116 [arXiv:1109.4280] [INSPIRE].

[25] D.S. Berman and M.J. Perry, Generalized geometry and M-theory, JHEP 06 (2011) 074 [arXiv: 1008.1763] [INSPIRE].

[26] D.S. Berman, H. Godazgar, M.J. Perry and P. West, Duality invariant actions and generalised geometry, JHEP 02 (2012) 108 [arXiv:1111.0459] [INSPIRE].

[27] D.S. Berman, H. Godazgar, M. Godazgar and M.J. Perry, The local symmetries of M-theory and their formulation in generalised geometry, JHEP 01 (2012) 012 [arXiv:1110.3930] [INSPIRE].

[28] H. Godazgar, M. Godazgar and M.J. Perry, E $E_{8}$ duality and dual gravity, JHEP 06 (2013) 044 [arXiv: 1303.2035] [INSPIRE].

[29] C. Strickland-Constable, Subsectors, Dynkin diagrams and new generalised geometries, arXiv: 1310.4196 [INSPIRE]. 
[30] H. Samtleben and M. Weidner, Gauging hidden symmetries in two dimensions, JHEP 08 (2007) 076 [arXiv:0705.2606] [INSPIRE].

[31] P.C. West, $E_{11}$ and M-theory, Class. Quant. Grav. 18 (2001) 4443 [hep-th/0104081] [INSPIRE].

[32] F. Riccioni and P.C. West, The $E_{11}$ origin of all maximal supergravities, JHEP 07 (2007) 063 [arXiv:0705.0752] [INSPIRE].

[33] E.A. Bergshoeff, I. De Baetselier and T.A. Nutma, $E_{11}$ and the embedding tensor, JHEP 09 (2007) 047 [arXiv:0705.1304] [InSPIRE].

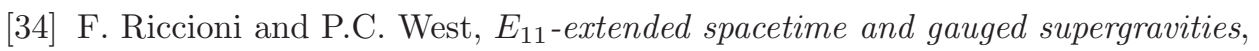
JHEP 02 (2008) 039 [arXiv:0712.1795] [INSPIRE].

[35] P. West, Generalised geometry, eleven dimensions and E $E_{11}$, JHEP 02 (2012) 018 [arXiv:1111.1642] [INSPIRE].

[36] P. West, $E_{11}$, generalised space-time and equations of motion in four dimensions, JHEP 12 (2012) 068 [arXiv: 1206.7045] [INSPIRE].

[37] F. Riccioni, D. Steele and P. West, The $E_{11}$ origin of all maximal supergravities: the hierarchy of field-strengths, JHEP 09 (2009) 095 [arXiv: 0906.1177] [INSPIRE].

[38] J. Palmkvist, Tensor hierarchies, Borcherds algebras and E $E_{11}$, JHEP 02 (2012) 066 [arXiv: 1110.4892] [INSPIRE].

[39] J. Palmkvist, The tensor hierarchy algebra, J. Math. Phys. 55 (2014) 011701 [arXiv: 1305.0018] [INSPIRE].

[40] J. Greitz, P. Howe and J. Palmkvist, The tensor hierarchy simplified, arXiv:1308.4972 [INSPIRE].

[41] M. Henneaux, B.L. Julia and J. Levie, $E_{11}$, Borcherds algebras and maximal supergravity, JHEP 04 (2012) 078 [arXiv: 1007.5241] [INSPIRE].

[42] D.S. Berman, E.T. Musaev, D.C. Thompson and D.C. Thompson, Duality invariant M-theory: gauged supergravities and Scherk-Schwarz reductions, JHEP 10 (2012) 174 [arXiv: 1208.0020] [INSPIRE].

[43] E.T. Musaev, Gauged supergravities in 5 and 6 dimensions from generalised Scherk-Schwarz reductions, JHEP 05 (2013) 161 [arXiv:1301.0467] [INSPIRE].

[44] G. Aldazabal, M. Graña, D. Marqués and J. Rosabal, Extended geometry and gauged maximal supergravity, JHEP 06 (2013) 046 [arXiv:1302.5419] [INSPIRE].

[45] J.-H. Park and Y. Suh, U-geometry: SL(5), JHEP 04 (2013) 147 [arXiv:1302.1652] [INSPIRE].

[46] M. Cederwall, J. Edlund and A. Karlsson, Exceptional geometry and tensor fields, JHEP 07 (2013) 028 [arXiv: 1302.6736] [INSPIRE].

[47] C.D. Blair, E. Malek and J.-H. Park, M-theory and type IIB from a duality manifest action, JHEP 01 (2014) 172 [arXiv:1311.5109] [INSPIRE].

[48] O. Hohm and H. Samtleben, Gauge theory of Kaluza-Klein and winding modes, Phys. Rev. D 88 (2013) 085005 [arXiv:1307.0039] [INSPIRE].

[49] O. Hohm and H. Samtleben, U-duality covariant gravity, JHEP 09 (2013) 080 [arXiv: 1307.0509] [INSPIRE]. 
[50] O. Hohm and H. Samtleben, Exceptional form of D = 11 supergravity, Phys. Rev. Lett. 111 (2013) 231601 [arXiv:1308.1673] [INSPIRE].

[51] O. Hohm and H. Samtleben, Exceptional field theory $I: E_{6(6)}$ covariant form of M-theory and type IIB, arXiv: 1312.0614 [INSPIRE].

[52] B. de Wit, H. Samtleben and M. Trigiante, The maximal $D=4$ supergravities, JHEP 06 (2007) 049 [arXiv:0705.2101] [INSPIRE].

[53] B. de Wit, H. Samtleben and M. Trigiante, On Lagrangians and gaugings of maximal supergravities, Nucl. Phys. B 655 (2003) 93 [hep-th/0212239] [INSPIRE].

[54] M. Weidner, Gauged supergravities in various spacetime dimensions, Fortsch. Phys. 55 (2007) 843 [hep-th/0702084] [INSPIRE].

[55] B. de Wit, H. Nicolai and H. Samtleben, Gauged supergravities, tensor hierarchies and M-theory, JHEP 02 (2008) 044 [arXiv:0801.1294] [INSPIRE].

[56] B. de Wit and H. Samtleben, The end of the p-form hierarchy, JHEP 08 (2008) 015 [arXiv: 0805.4767] [INSPIRE].

[57] B. de Wit and M. van Zalk, Supergravity and M-theory, Gen. Rel. Grav. 41 (2009) 757 [arXiv: 0901.4519] [INSPIRE].

[58] E.A. Bergshoeff, J. Hartong, O. Hohm, M. Huebscher and T. Ortín, Gauge theories, duality relations and the tensor hierarchy, JHEP 04 (2009) 123 [arXiv:0901.2054] [INSPIRE].

[59] C.M. Hull, U duality and BPS spectrum of super Yang-Mills theory and M-theory, JHEP 07 (1998) 018 [hep-th/9712075] [INSPIRE].

[60] E.A. Bergshoeff and F. Riccioni, The D-brane U-scan, arXiv:1109.1725 [INSPIRE].

[61] J. de Boer and M. Shigemori, Exotic branes in string theory, Phys. Rept. 532 (2013) 65 [arXiv: 1209.6056] [INSPIRE].

[62] M. Graña and D. Marques, Gauged double field theory, JHEP 04 (2012) 020 [arXiv: 1201.2924] [INSPIRE].

[63] D. Geissbuhler, D. Marques, C. Núñez and V. Penas, Exploring double field theory, JHEP 06 (2013) 101 [arXiv:1304.1472] [INSPIRE].

[64] G. Dibitetto, J. Fernandez-Melgarejo, D. Marques and D. Roest, Duality orbits of non-geometric fluxes, Fortsch. Phys. 60 (2012) 1123 [arXiv:1203.6562] [INSPIRE].

[65] O. Hohm and S.K. Kwak, Double field theory formulation of heterotic strings, JHEP 06 (2011) 096 [arXiv:1103.2136] [INSPIRE].

[66] D.S. Berman and K. Lee, Supersymmetry for gauged double field theory and generalised Scherk-Schwarz reductions, Nucl. Phys. B 881 (2014) 369 [arXiv:1305.2747] [INSPIRE].

[67] M. Graña and F. Orsi, $N=1$ vacua in exceptional generalized geometry, JHEP 08 (2011) 109 [arXiv:1105.4855] [INSPIRE].

[68] G. Dall'Agata, G. Inverso and M. Trigiante, Evidence for a family of $\mathrm{SO}(8)$ gauged supergravity theories, Phys. Rev. Lett. 109 (2012) 201301 [arXiv:1209.0760] [inSPIRE]. 